\title{
Dynamic Problems of the Planets and Asteroids, and Their Discussion
}

\author{
Joseph J. Smulsky ${ }^{1^{*}}$, Yaroslav J. Smulsky² \\ ${ }^{1}$ Institute of the Earth's Cryosphere, Siberian Branch of Russian Academy of Sciences, Tyumen, Russia \\ ${ }^{2}$ Institute of Thermophysics of Russian Academy of Sciences, Siberian Branch, Novosibirsk, Russia \\ Email: ${ }^{*}$ jsmulsky@mail.ru, ysmulskii@mail.ru
}

Received February 9, 2012; revised March 15, 2012; accepted April 18, 2012

\begin{abstract}
The problems of dynamics of celestial bodies are considered which in the literature are explained by instability and randomness of movements. The dynamics of planets orbits on an interval 100 million years was investigated by new numerical method and its stability is established. The same method is used for computing movements of two asteroids Apophis and 1950 DA. The evolution of their movement on an interval of 1000 is investigated. The moments of their closest passages at the Earth are defined. The different ways of transformation of asteroids trajectories into orbits of the Earth's satellites are considered. The problems of interest are discussed from the different points of view.
\end{abstract}

Keywords: Dynamics; Planets; Asteroids; Satellites; Stability; Discussion

\section{Introduction}

In the last decades a number of problems associated with the precision of calculating movements have accumulated in the celestial and the space dynamics. It was found that there were discrepancies between the calculated and the observed movements. These differences were the cause of the conclusions about chaotic movements and the impossibility of accurately calculating them. In addition to Newton's gravitational forces were introduced weaker forces of other nature, as well as new substances "dark energy" and "dark matter". In this paper we consider the problems that are associated only with the dynamics of the two groups of celestial objects: With planets and asteroids.

In the study of the evolution of the Solar system over geological time scales the number of researchers has come to the conclusion that there are the instability of the planetary orbits and chaotic motions in the Solar system. For example, in paper [1], it is noted that the eccentricity of the orbit of Mars can be greater than 0.2, and chaotic diffusion of Mercury is so great that his eccentricity can potentially reach values close to 1 and the planet could be thrown out of the Solar system. Already at the time intervals of $10 \mathrm{Myr}$ (million years) there is the weak divergence of the Earth's orbit [2], which, according to these authors, is caused by multiple resonances in the Solar system. Due to them the movement of Solar system is chaotic. Therefore the motion of the Earth [2] and

"Corresponding author.
Mars [3] with an acceptable accuracy cannot be calculated for a time greater than $20 \mathrm{Myr}$.

The same problems of dynamics are occurred in the study of motion of asteroids. Unlike the planets, their movement is considered on a smaller time intervals. However, the higher accuracy of determination of their movement is required. In connection with the urgency of the tasks of asteroids motion the problems of their dynamics is considered in more detail.

Over the past decade, the asteroids of prime interest have been two asteroids, Apophis and 1950 DA, the first predicted to approach the Earth in 2029, and the second, in 2880. Reported calculations revealed some probability of an impact of the asteroids on the Earth. Yet, by the end of the decade refined orbital-element values of the asteroids were obtained, and more precise algorithms for calculating the interactions among solar-system bodies were developed. Following this, in the present paper we consider the motion evolution of both asteroids. In addition, we discuss available possibilities for making the asteroids into the Earth-bound satellites. Initially, the analysis is applied to Apophis and, then, numerical data for 1950 DA obtained by the same method will be presented.

The background behind the problem we treat in the present study was recently outlined in [4]. On June 19-20, 2004, asteroid Apophis was discovered by astronomers at the Kitt Peak Observatory [5], and on December 20, 2004 this asteroid was observed for the second time by astronomers from the Siding Spring Survey Observato- 
ry [6]. Since then, the new asteroid has command international attention. First gained data on identification of Apophis' orbital elements were employed to predict the Apophis path. Following the first estimates, it was reported in [7] that on April 13, 2029 Apophis will approach the Earth center to a minimum distance of 38,000 $\mathrm{km}$. As a result of the Earth gravity, the Apophis orbit will alter appreciably. Unfortunately, presently available methods for predicting the travel path of extraterrestrial objects lack sufficient accuracy, and some authors have therefore delivered an opinion that the Apophis trajectory will for long remain unknown, indeterministic, and even chaotic (see $[4,7,8]$ ). Different statistical predictions points to some probability of Apophis' collision with the Earth on April 13, 2036. It is this aspect, the impact risk, which has attracted primary attention of workers dealing with the problem.

Rykhlova et al. [7] have attempted an investigation into the possibility of an event that the Apophis will closely approach the Earth. They also tried to evaluate possible threats stemming from this event. Various means to resist the fall of the asteroid onto Earth were put forward, and proposals for tracking Apophis missions, made. Finally, the need for prognostication studies of the Apophis path accurate to a one-kilometer distance for a period till 2029 was pointed out.

Many points concerning the prospects for tracking the Apophis motion with ground- and space-based observing means were discussed in [4,7-9]. Since the orbits of the asteroid and Earth pass close to each other, then over a considerable portion of the Apophis orbit the asteroid disc will only be partially shined or even hidden from view. That is why it seems highly desirable to identify those periods during which the asteroid will appear accessible for observations with ground means. In using space-based observation means, a most efficient orbital allocation of such means needs to be identified.

Prediction of an asteroid motion presents a most challenging problem in astrodynamics. In paper [10], the differential equations for the perturbed motion of the asteroid were integrated by the Everhart method [11]; in those calculations, for the coordinates of perturbing bodies were used the JPL planetary ephemeris DE403 and DE405 issued by the Jet Propulsion Laboratory, USA. Sufficient attention was paid to resonance phenomena that might cause the hypothetical 2036 Earth impact.

Bykova and Galushina [12,13] used 933 observations to improve the identification accuracy for initial Apophis orbital parameters. Yet, the routine analysis has showed that, as a result of the pass of the asteroid through several resonances with Earth and Mars, the motion of the asteroid will probably become chaotic. With the aim to evaluate the probability of an event that Apophis will impact the Earth in 2036, Bykova et al. [12] have made about 10 thousand variations of initial conditions, 13 of which proved to inflict a fall of Apophis onto Earth.

Smirnov [14] has attempted a test of various integration methods for evaluating their capabilities in predicting the motion of an asteroid that might impact the Earth. The Everhart method, the Runge-Kutta method of fourth order, the Yoshida methods of sixth and eighth orders, the Hermit method of fourth and sixth orders, the Multistep Predictor-Corrector (MS-PC) method of sixth and eighth orders, and the Parker-Sochacki method were analyzed. The Everhart and MS-PC methods proved to be less appropriate than the other methods. For example, at close Apophis-to-Earth distances Smirnov [14] used, instead of the Everhart method, the Runge-Kutta method. He came to the fact that, in the problems with singular points, finite-difference methods normally fail to accurately approximate higher-order derivatives. This conclusion is quite significant since below we will report on an integration method for motion equations free of such deficiencies.

In paper [15] the mathematical problems on asteroid orbit prediction and modification were considered. Possibilities offered by the impact-kinetic and thermonuclear methods in correcting the Apophis trajectory were evaluated.

An in-depth study of the asteroid was reported in paper [4]. A chronologically arranged outline of observational history was given, and the trend with progressively reduced uncertainty region for Apophis' orbit-element values was traced. Much attention was paid to discussing the orbit prediction accuracy and the bias of various factors affecting this accuracy. The influence of uncertainty in planet coordinates and in the physical characteristics of the asteroid, and also the perturbing action of other asteroids, were analyzed. The effects on integration accuracy of digital length, non-spherical shape of Earth and Moon, solar-radiation-induced perturbations, non-uniform thermal heating, and other factors, were examined.

The equations of perturbed motion of the asteroid were integrated with the help of the Standard Dynamic Model (SDM), with the coordinates of other bodies taken from the JPL planetary ephemeris DE405. It is a well-known fact that the DE405 ephemerid was compiled as an approximation to some hundred thousand observations that were made till 1998. Following the passage to the ephemeris DE414, that approximates observational data till 2006, the error in predicting the Apophis trajectory on 2036 has decreased by 140,000 km. According to Giorgini et al. [4], this error proved to be ten times greater than the errors induced by minor perturbations. Note that this result points to the necessity of employing a more accurate method for predicting the asteroid path.

In paper [4], prospects for further refinement of Apophis' trajectory were discussed at length. Time periods 
suitable for optical and radar measurements, and also observational programs for oppositions with Earth in 2021 and 2029 and spacecraft missions for 2018 and 2027 were scheduled. Future advances in error minimization for asteroid trajectory due to the above activities were evaluated.

It should be noted that the ephemerides generated as an approximation to observational data enable rather accurate determination of a body's coordinates in space within the approximation time interval. The prediction accuracy for the coordinates on a moment remote from this interval worsens, the worsening being the greater the more the moment is distant from the approximation interval. Therefore, the observations and the missions scheduled in paper [4] will be used in refining future ephemerides.

In view of the afore-said, in calculating the Apophis trajectory the equation of perturbed motion were integrated $[4,10,15]$, while the coordinates of other bodies were borrowed from the ephemerid. Difference integration methods were employed, which for closely spaced bodies yield considerable inaccuracies in calculating higher-order derivatives. Addition of minor interactions to the basic Newtonian gravitational action complicates the problem and enlarges the uncertainty region in predicting the asteroid trajectory. Many of the weak interactions lack sufficient quantitative substantiation. Moreover, the physical characteristics of the asteroid and the interaction constants are known to some accuracy. That is why in making allowance for minor interactions expert judgments were used. And, which is most significant, the error in solving the problem on asteroid motion with Newtonian interaction is several orders greater than the corrections due to weak additional interactions.

The researches, for example, Bykova and Galushina $[12,13]$ apply a technique in Giorgini et al. 2008 to study of influence of the initial conditions on probability of collision Apophis with Earth. The initial conditions for asteroid are defined from elements of its orbit, which are known with some uncertainty. For example, eccentricity value $e=e_{n} \pm \sigma_{e}$, where $e_{n}$ is nominal value of eccentricity, and $\sigma_{e}$ is root-mean-square deviation at processing of several hundred observation of asteroid. The collision parameters are searched in the field of possible motions of asteroid, for example for eccentricity, $3 \sigma_{e}$, the initial conditions are calculated in area $e=e_{n} \pm \sigma_{e}$. From this area the 10 thousand, and in some works, the 100 thousand sets of the initial conditions are chosen by an accidental manner, i.e. instead of one asteroid it is considered movement 10 or 100 thousand asteroids. Some of them can come in collision with Earth. The probability of collision asteroid with the Earth is defined by their amount.

Such statistical direction is incorrect. If many mea- surement data for a parameter are available, then the nominal value of the parameter, say, eccentricity $e_{n}$, presents a most reliable value for it. That is why a trajectory calculated from nominal initial conditions can be regarded as a most reliable trajectory. A trajectory calculated with a small deviation from the nominal initial conditions is a less probable trajectory, whereas the probability of a trajectory calculated from the parameters taken at the boundary of the probability region (i.e. from $e=e_{n} \pm \sigma_{e}$ ) tends to zero. Next, a trajectory with initial conditions determined using parameter values trice greater than the probable deviations (i.e. $e=e_{n} \pm 3 \sigma_{e}$ ) has an even lower, negative, probability. Since initial conditions are defined by six orbital elements, then simultaneous realization of extreme (boundary) values $( \pm 3 \sigma)$ for all elements is even a less probable event, i.e. the probability becomes of smaller zero.

That is why it seems that a reasonable strategy could consist in examining the effect due to initial conditions using such datasets that were obtained as a result of successive accumulation of observation data. Provided that the difference between the asteroid motions in the last two datasets is insignificant over some interval before some date, it can be concluded that until this date the asteroid motion with the initial conditions was determined quite reliably.

As it was shown in paper [4], some additional activeties are required, aimed at further refinement of Apophis' trajectory. In this connection, more accurate determination of Apophis' trajectory is of obvious interest since, following such a determination, the range of possible alternatives would diminish.

For integration of differential motion equations of solar-system bodies over an extended time interval, a program Galactica was developed [16-19]. In this program, only the Newtonian gravity force was taken into account, and no differences for calculating derivatives were used. In the problems for the compound model of Earth rotation [20] and for the gravity maneuver near Venus [21], motion equations with small body-to-body distances, the order of planet radius, were integrated. Following the solution of those problems and subsequent numerous checks of numerical data, we have established that, with the program Galactica, we were able to rather accurately predict the Apophis motion over its travel path prior to and after the approach to the Earth. In view of this, in the present study we have attempted an investigation into orbit evolution of asteroids Apophis and 1950 DA; as a result of this investigation, some fresh prospects toward possible use of these asteroids have opened.

\section{Problem Statement}

For the asteroid, the Sun, the planets, and the Moon, all interacting with one another by the Newton law of gra- 
vity, the differential motion equations have the form [16]:

$$
\frac{\mathrm{d}^{2} \boldsymbol{r}_{i}}{\mathrm{~d} t^{2}}=-G \sum_{k \neq i}^{n} \frac{m_{k} \boldsymbol{r}_{i k}}{r_{i k}{ }^{3}}, i=1,2, \cdots, n
$$

where $\boldsymbol{r}_{i}$ is radius-vector of a body with mass $m_{i}$ relatively Solar System barycenter; $G$ is gravitational constant; $\boldsymbol{r}_{i k}$ is vector $\boldsymbol{r}_{i}-\boldsymbol{r}_{k}$ and $r_{i k}$ is its module; $n=12$.

As a result of numerical experiments and their analysis we came to a conclusion, that finite-difference methods of integration do not provide necessary accuracy. For the integration of Equation (1) we have developed algorithm and program Galactica. The meaning of function at the following moment of time $t=t_{0}+\Delta t$ is determined with the help of Taylor series, which, for example, for coordinate $x$ looks like:

$$
x=x_{0}+\sum_{k=1}^{K} \frac{1}{k !} x_{0}^{(k)}(\Delta t)^{k}
$$

where $x_{0}^{(k)}$ is derivative of $k$ order at the initial moment $t_{0}$.

The meaning of velocity $x^{\prime}$ is defined by the similar formula, and acceleration $x_{0}^{\prime \prime}$ by the Equation (1). Higher derivatives $x_{0}^{(k)}$ we are determined analytically as a result of differentiation of the Equation (1). The calculation algorithm of the sixth order is now used, i.e. with $K=6$.

A few words about the method used and the program Galactica. The algorithms of finite-difference methods are derived from the Taylor series (2). In this case the higher derivatives are determined by the difference of the second derivatives at different steps. This leads to errors of integration. There are methods [22,23], in which the derivatives are determined by recurrence formulas. In the program Galactica the derivatives are calculated under the exact analytical formulas, which we have deduced. This provides greater accuracy than other methods.

Besides in the program Galactica there are many other details, which allow to not reducing the achieved in such way accuracy. They were found in the process of creating the program Galactica. During its development more than 10 different ways to control errors has been used. In our book [19] the following checks are made mention of:

1) Checking the stability of the angular momentum $M$ of the Solar System;

2) Checking the magnitude of the momentum $P$ of the Solar System;

4) Integrating backwards and forwards in time;

5) Integrating into a remote epoch and subsequent return to the initial epoch;

6) Picking persistent changes (orbital major semiaxis, period, or precession axis etc.) and their checking;

7) Checking against test problems with exact analyticcal solutions, for example, $n$-axisymmetrical problem [24];
8) Comparison with observations;

9) Comparison with other reported data.

The high accuracy of the program Galactica is firstly allowed to integrate Equations (1) for the motion of the Solar System for 100 million years [19]. The error was less on some orders in comparison with work [25], in which this problem has been solved for 3 million years by Stórmer method.

The program Galactica allows solving the problem of interaction of any number of bodies, which motion is described by Equations (1). For example, the problem of the evolution of Earth's rotation axis [20] was solved. In this task, the rotational motion of the Earth's has been replaced by a compound model of the Earth's rotation. The compound model of the Sun's rotation was used in another task [26], in which the influence of the oblate rotating Sun on the motion of planets was established. In all these problems, this method of integration and program Galactica had no failures and we successfully used them.

As noted above, the methods, which use Standard Dynamic Model, are approximation of the data of Solar System body's observations. To calculate the future movement of the asteroid the bodies' positions are used outside the framework of observation. Therefore the calculation error increases with distance in time from the base of observations. The base of observations is not used in program Galactica, so this error is missing in it. A high accuracy of the integration method of Galactica allows for a smaller error compute motion of asteroids in their rapprochement with the celestial bodies.

The free-access Galactica system, user version for personal computer, can be found at:

http://www.ikz.ru/ smulski/GalactcW. The system of Galactica, except the program Galactica, includes several components, which are described in the User's Guide http://www.ikz.ru/ smulski/GalactcW/GalDiscrE.pdf. The Guide also provides detailed instructions for all stages of solving the problem. After the free-access system is created based on a supercomputer, we will post the information at the above site.

\section{The Dynamics of the Planets' Orbits}

To study the dynamics of the Solar system we used the initial conditions (IC) on the 30.12.1949 with the Julian day $J D_{0}=2433280.5$ in two versions. The first version of the IC was based on ephemerid DE19 of Jet Propulsion Laboratory, USA (JPL), a second version is based on the ephemerid DE406. The first IC was in a coordinate system of 1950.0, while the second-in the system of 2000.0. These initial conditions are given in our paper [19] and on the website http://www.ikz.ru/ smulski/Data/ OrbtData. As already mentioned, the differential Equa- 
tions (1) were integrated for 11 bodies: 9 planets, the Sun and the Moon. The motion of bodies is considered in barycentric equatorial coordinates. The parameters of the orbits of the planets are defined in the heliocentric coordinate system and the orbit of the Moon is in geocentric coordinates.

The main results obtained with the integration step $\Delta t=$ $10^{-4}$ year and the number of double length (17 decimal). Checking and clarifying the calculations were performed with a smaller step, as well as with the extended length of the numbers (up to 34 decimal places).

Methods for validation of the solutions and their errors are investigated in [19]. The computed with the help of the program Galactica change of the parameters of the orbit of Mars at time interval of 7 thousand years is shown in Figure 1 points 1. The eccentricity $e$ and perihelion angle $\varphi_{p}$ increase, and the longitude of the ascending node $\varphi_{\Omega}$ decreases in this span of time. The angle of inclination of the Mars orbit $i$ does not change monotonically. In the epoch at $T=1400$ years from 30 December 1949, it has a maximum value. The changing these parameters for a century are called secular perturbations. In contrast, the semi-major axis $a$ and orbital period $P$ on the average remains unchanged, so the graphs are given their deviations from the mean values. These fluctuations relative to the average $a_{m}=2.279 \times$ $10^{11} \mathrm{~m}$ and $P_{m}=1.881$ years have a small value. The parameters $e, i, \varphi_{\Omega}$ and $\varphi_{p}$ also vary with the same relative amplitudes as parameters $a$ and $P$.

In Figure 1 the lines 2 and 3 show the approximation of the observation data. As one can see, the eccentricity $e$ and the angles $i, \varphi_{\Omega}$ and $\varphi_{p}$ coincide with observations in the interval \pm 1000 years, i.e. within the validity of approximations by S. Newcomb [27] and J. Simon et al. [28]. Calculations for the semi-major axis $a$ and the periods $P$ also coincide with the observations and their fluctuations comparable in magnitude with the difference between the approximations of different authors. Similar studies of secular changes in orbital parameters are made for other planets. They are also compared with the approximations of the observational data, except for Pluto. Its approximation of the observations is missing.

In Figure 2 there are the variations of the calculated elements of the orbit of Mars in 3 million years into the past. The eccentricity of the orbit $e$ has short-period oscillations of amplitude 0.019 with the main period equal to $T_{e 1}=95.2$ thousand years. It oscillates relatively the average for 50 million years value of $e_{m}=0.066$. The longitude of ascending node $\varphi_{\Omega}$ oscillates with the average period of $T_{\Omega}=73.1$ thousand years around the mean value $\varphi_{\Omega m}=0.068$ radians. The angle of inclination of the orbital plane to the equatorial plane $i$ fluctuates with the same period $T_{i}=73.1$ thousand years around the mean value $i_{m}=0.405$ radians. Longitude of perihelion $\varphi_{p}$ al-

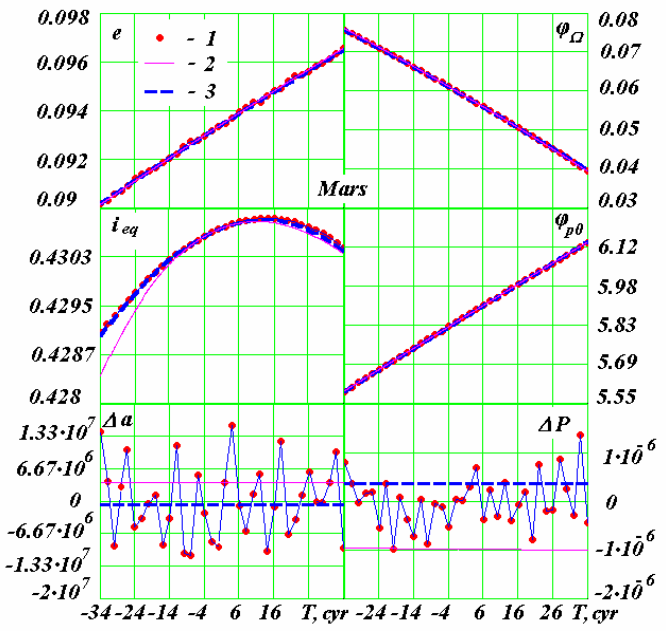

Figure 1. Secular changes of the Mars orbit 1 compared with approximated observations by Newcomb [27] and Simon et al. [28], 2 and 3, respectively: Eccentricity $e$; inclination $i$ to equator plane for epoch $\mathbf{J 2 0 0 0 . 0}$; longitude of ascending node $\varphi_{\Omega}$ relative to the $x$ axis at $\mathbf{J 2 0 0 0 . 0}$; longitude of perihelion $\varphi_{p}$; semi-major axis deviation $\Delta a$ from the average of 7 thousand years, in meters; orbital period deviation $\Delta P$ from average of 7 thousand years, in centuries. Angles are in radians and time $T$ is in centuries from 30 December 1949; data points spaced at $200 \mathrm{yr}$.

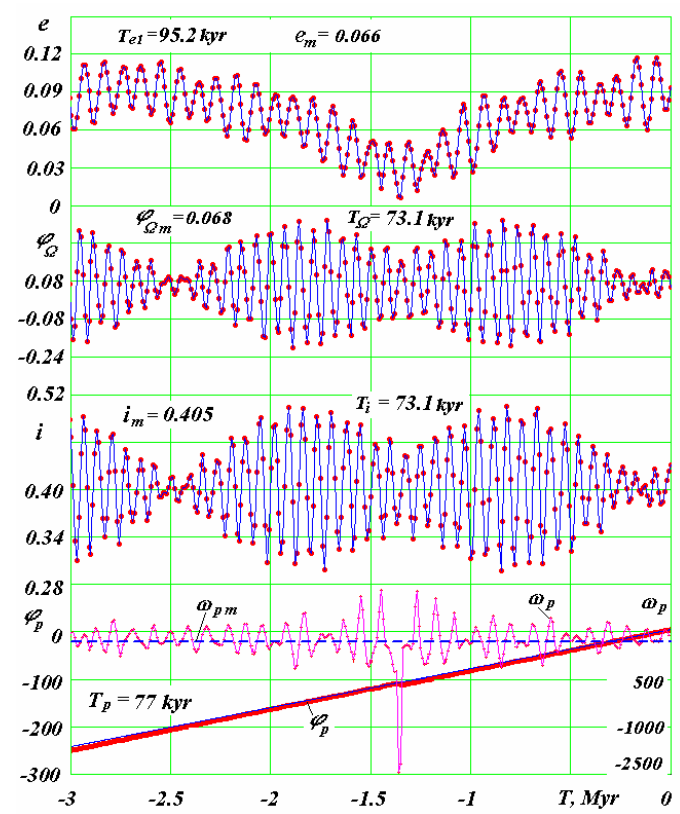

Figure 2. Evolution of the orbit parameters of Mars for 3 Myr. $\omega_{p}$ is the angular velocity of the perihelion rotation in the "century for the time interval of 20 thousand years: $\omega_{p m}=$ 1687 " century is the average angular velocity of perihelion rotation during 50 million years; $T_{e 1}, T_{\Omega}$ and $T_{i}$ are the shortest periods of eccentricity, ascending node, and inclination, respectively, in kyr (thousand years); $e_{m}, i_{m}$ and $\varphi_{\Omega m}$ are the average values of appropriate parameters; $T_{p}$ is a rotation perihelion period averaged over $50 \mathrm{Myr}$. Other notations see Figure 1. 
most linearly increases with time, i.e. the perihelion moves in the direction of circulation of Mars around the Sun, making on the average for a -50 million years, one revolution for the time $T_{p}=76.8$ thousand years. At the same time its motion is uneven. As one can see from the graph, the angular velocity of rotation of the perihelion $\omega_{p}$ fluctuates around the average value of $\omega_{p m}=1687 "$ (arc-seconds) per century, while at time $T=-1.35$ million years, it takes a large negative value, i.e. in this moment there is the return motion of the perihelion with great velocity.

As a result of research for several million years the periods and amplitudes of all fluctuations of the orbits parameters of all planets received. The system of Equation (1) with the help of the program Galactica was integrated for 100 million years in the past and the evolution of the orbits of all planets and the Moon was studied. Figure 3 shows changing parameters of the Mars orbit on an interval from -50 million years to -100 million years. The eccentricity $e$, angles of inclination $i$, ascending node $\varphi_{\Omega}$ monotonically oscillate. The fluctuations have several periods, and duration of most of them is much smaller than the interval in 50 million years. For example, the greatest period eccentricity $T_{e 2}=2.31$ million years. The angular velocity $\omega_{p}$ of perihelion rotation fluctuate around the average value of $\omega_{p m}$. In comparison with the

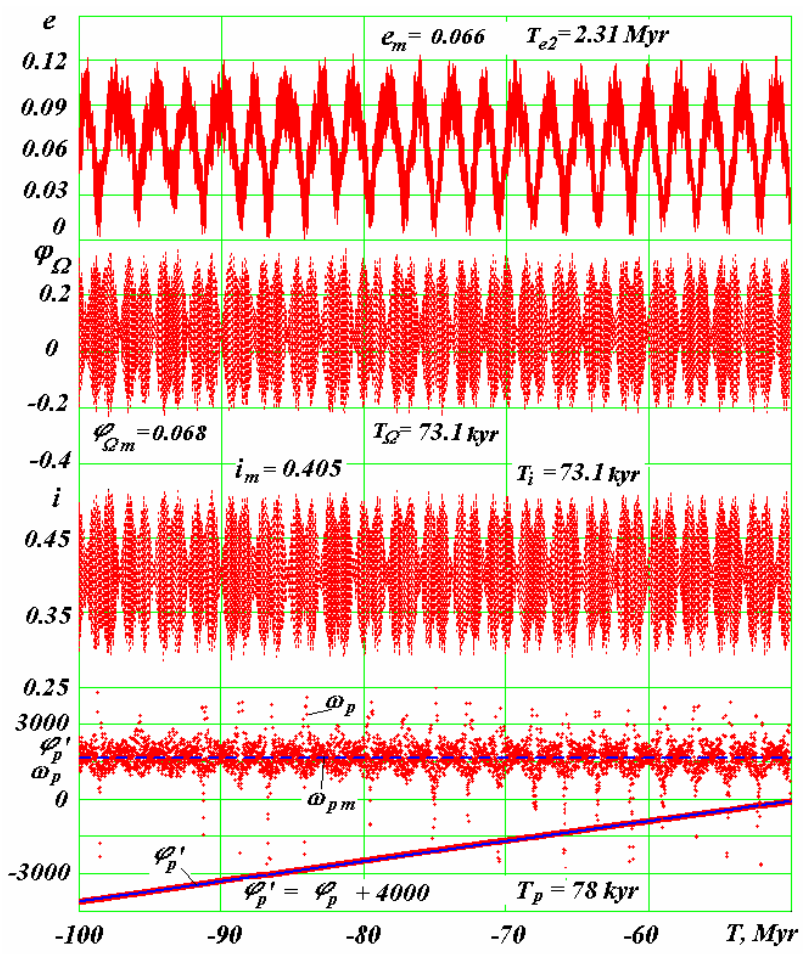

Figure 3. The evolution of the Mars orbit in the second half of the period of 100 million years: $T$ is the time in millions of years into the past from the epoch 30.12.1949; other notations see Figures 1 and 2. eccentricity $e$ one can see that negative values of $\omega_{p}$, i.e. reciprocal motion of the perihelion occurs when the eccentricity of the orbit is close to zero.

On the interval from 0 to -50 million years the graphics have the same form [19], as shown in Figure 3, i.e. the orbit of Mars is both steady and stable and there is no tendency to its change. Similar results were obtained for other planets, i.e. these studies established the stability of the orbits and the Solar system as a whole. The result is important, since in the abovementioned papers [1-3] at solving the problem of other methods after 20 million years, the orbit begins to change, what led to the destruction of the Solar system in the future. Based on these solutions their authors came to the conclusion about the instability of the Solar system and chaotic motions in it.

During these researches we have established, that the evolution of the planets orbits is the result of four movements: 1) The precession of the orbital axis $\boldsymbol{S}$ around a fixed in space vector of angular momentum $\boldsymbol{M}$ of the Solar system; 2) Nutational oscillations of the orbit axis $\boldsymbol{S} ; 3)$ The oscillations of orbit; 4) the rotation of the orbit in its own plane (rotation of the perihelion). The behavior of the perihelion of the orbits of all planets in the span of 50 million years is shown in Figure $\mathbf{4}$ as a function of angles perihelion $\varphi_{p}$ versus time. The orbits of the eight planets from Mercury to Neptune are orbiting counterclockwise, i.e. in the direction of orbital motion. The orbit of Pluto, the only one, that rotates clockwise. Two

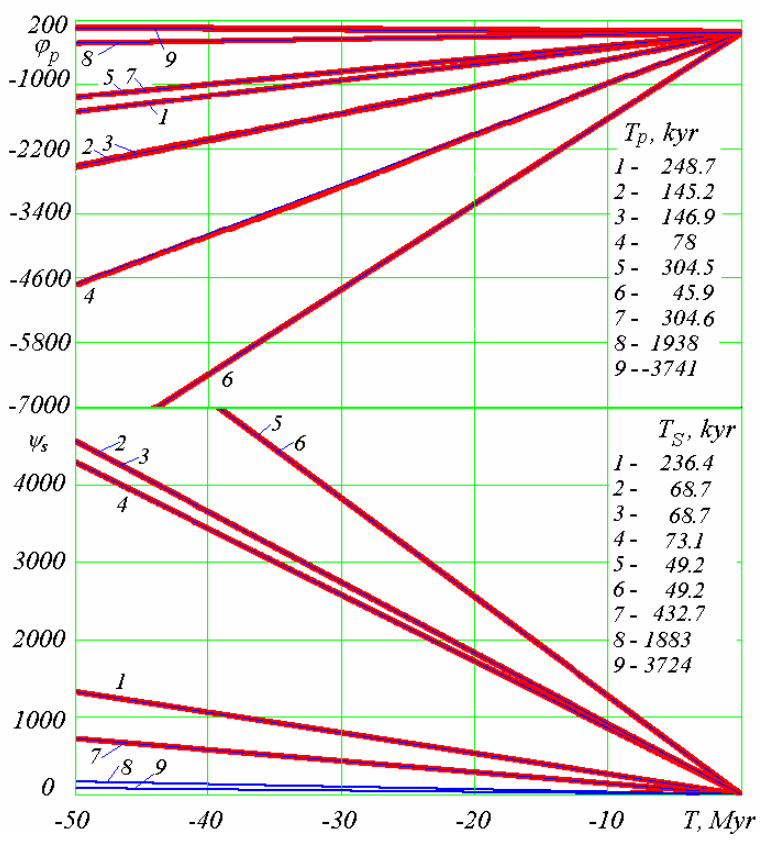

Figure 4. Variations of perihelion longitudes $\varphi_{p}$ and the precession angle $\psi_{S}$ for $50 \mathrm{Myr}$ in orbits of nine planets from Mercury to Pluto (numbered from 1 to 9), with the respective perihelion periods $\left(T_{p}\right)$ and precession cycles $\left(T_{S}\right)$ in Kyr, which averaged over 50 Myr. 
groups of planets (Venus and Earth, Jupiter and Uranus), as seen from the values of the periods $T_{p}$, have almost the same velocity of perihelion rotation. The orbit of Saturn has the highest velocity, and the orbit of Pluto has the smallest one.

The precession of the orbit occurs clockwise, i.e. against the orbital motion of the planets The angle of precession $\psi_{S}$ of the orbit axis is defined in the plane perpendicular to the vector of the momentum $\boldsymbol{M}$. This plane crosses the equator plane on the line directed at an angle $\varphi_{\Omega m}=0.068$ to the $x$ axis. Precession angle $\psi_{S}$ is the angle between the line of intersection and the node of the planet orbit. The angle $\psi_{S}$, as the angle of the perihelion $\varphi_{p}$, varies irregularly, but a large time interval of 50 million years, as shown in Figure 4, these irregularrities are not visible. As can be seen from the periods of $T_{S}$, the orbits axis of Jupiter and Saturn precess with maximum velocity and Pluto-with the lowest one. For the two groups of planets: Venus and Earth, Jupiter and Saturn, the velocity of precession is practically the same.

It should be emphasized that the secular changes of angles of inclination $i$ and ascending node $\varphi_{\Omega}$ in Figure 1 and their variations, are presented by the graphics in Figures 2 and 3 , are due to the precessional and nutational motion of the orbit axis $\boldsymbol{S}$.

The studies testify that the evolution of the planets orbits in the investigated range is unchanged and stable. This allows us to conclude that the manifestations of instability and chaos in the motion of the planets, as described by other authors, most likely due to the methods of solution which they have been used. Furthermore, it should also be emphasized that reported in literature resonances and instabilities appear in the simplified equations of motion when they are solved by approximate analytical methods. These phenomena do not arise at the integrating not simplified Equation (1) by method (2).

\section{Preparation of Initial Data of Asteroids}

We consider the motion of asteroids in the barycentric coordinate system on epoch $\mathrm{J} 2000.0$, Julian day $J D_{s}=$ 2451545. The orbital elements asteroids Apophis and 1950 DA, such as the eccentricity $e$, the semi-major axis $a$, the ecliptic obliquity $i_{e}$, the ascending node angle $\Omega$, the ascending node-perihelion angle $\omega_{e}$, etc., and asteroids position elements, such as the mean anomaly $M$, were borrowed from the JPL Small-Body database 2008 as specified on November 30, 2008. The data, represented to 16 decimal digits, are given in Table 1.

For Apophis in Table 1 the three variants are given. The first variant is now considered. These elements correspond to the solution with number JPL sol. 140, which is received Otto Mattic at April 4, 2008. In Table 1 the uncertainties of these data are too given. The relative uncertainty value $\delta$ is in the range from $2.4 \times 10^{-8}$ to $8 \times$ $10^{-7}$. The same data are in the asteroid database by Edward Bowell [29], although these data are represented only to 8 decimal digits, and they differ from the former data in the 7-th digit, i.e., within value $\delta$. Giorgini et al. [4] used the orbital elements of Apophis on epoch $J D=$ 2453979.5 (September 01, 2006), which correspond to the solution JPL sol.142. On publicly accessible JPL-system

Table 1. Three variants of orbital elements of asteroids Apophis on two epochs and 1950 DA on one epoch in the heliocentric ecliptic coordinate system of 2000.0 with $J D_{S}=2451545$ (see JPL Small-Body Database [30]).

\begin{tabular}{|c|c|c|c|c|c|c|}
\hline \multirow{3}{*}{ Elements } & \multicolumn{4}{|c|}{ Apophis } & $1950 \mathrm{DA}$ & \multirow{3}{*}{ Units } \\
\hline & $\begin{array}{c}\text { 1-st variant } \\
\text { November } 30,2008 \\
J D_{01}=2454800.5 \\
\text { JPL sol.140 }\end{array}$ & $\begin{array}{c}\text { Uncertainties } \\
\pm \sigma \\
\text { 1-st var. }\end{array}$ & $\begin{array}{c}\text { 2-nd variant } \\
\text { January } 04,2010 \\
J D_{02}=2455200.5 \\
\text { JPL sol.144 }\end{array}$ & $\begin{array}{c}\text { 3-rd variant } \\
\text { November } 30,2008 \\
J D_{01}=2454800.5 \\
\text { JPL sol.144. }\end{array}$ & $\begin{array}{c}\text { November } 30,2008 \\
J D_{0}=2454800.5 \\
\text { JPL sol.51 }\end{array}$ & \\
\hline & \multicolumn{5}{|c|}{ Magnitude } & \\
\hline$e$ & 0.1912119299890948 & $7.6088 \mathrm{e}-08$ & 0.1912110604804485 & 0.1912119566344382 & 0.507531465407232 & \\
\hline$a$ & 0.9224221637574083 & $2.3583 \mathrm{e}-08$ & 0.9224192977379344 & 0.9224221602386669 & 1.698749639795436 & $\mathrm{AU}$ \\
\hline$q$ & 0.7460440415606373 & $8.6487 \mathrm{e}-08$ & 0.7460425256098334 & 0.7460440141364661 & 0.836580745750051 & $\mathrm{AU}$ \\
\hline$i_{e}$ & 3.331425002325445 & $2.024 \mathrm{e}-06$ & 3.331517779979046 & 3.331430909298658 & 12.18197361251942 & deg \\
\hline$\Omega$ & 204.4451349657969 & 0.00010721 & 204.4393039605681 & 204.4453098275707 & 356.782588306221 & deg \\
\hline$\omega_{e}$ & 126.4064496795719 & 0.00010632 & 126.4244705298442 & 126.4062862564680 & 224.5335527346193 & deg \\
\hline$M$ & 254.9635275775066 & $5.7035 \mathrm{e}-05$ & 339.9486156711335 & 254.9635223452623 & 161.0594270670401 & deg \\
\hline$t_{p}$ & $\begin{array}{c}2454894.912750123770 \\
(2009-M a r-04.41275013)\end{array}$ & $5.4824 \mathrm{e}-05$ & $\begin{array}{l}2455218.523239657948 \\
\text { (2010-Jan-22.02323966) }\end{array}$ & $\begin{array}{c}2454894.912754286546 \\
(2009-M a r-04.41275429)\end{array}$ & $\begin{array}{c}2.454438 .693685309 \\
\text { (2007-Dec-12.0419368531 }\end{array}$ & $\begin{array}{l}\mathrm{JD} \\
\mathrm{d}\end{array}$ \\
\hline$P$ & $\begin{array}{c}323.5884570441701 \\
0.89\end{array}$ & $\begin{array}{l}1.2409 \mathrm{e}-05 \\
3.397 \mathrm{e}-08\end{array}$ & $\begin{array}{c}323.5869489330219 \\
0.89\end{array}$ & $\begin{array}{c}323.5884551925927 \\
0.89\end{array}$ & $\begin{array}{c}808.7094041052905 \\
2.21\end{array}$ & $\begin{array}{l}\text { D } \\
\mathrm{yr}\end{array}$ \\
\hline$n$ & 1.112524233059586 & $4.2665 \mathrm{e}-08$ & 1.112529418096263 & 1.112524239425464 & 0.445153720449539 & $\operatorname{deg} / \mathrm{d}$ \\
\hline$Q$ & 1.098800285954179 & $2.8092 \mathrm{e}-08$ & 1.098796069866035 & 1.098800306340868 & 2.560918533840822 & $\mathrm{AU}$ \\
\hline
\end{tabular}


Horizons the solution sol.142 can be prolonged till November $30.0,2008$. In this case it is seen, that difference of orbital elements of the solution 142 from the solution 140 does not exceed $0.5 \sigma$ uncertainties of the orbit elements.

The element values in Table 1 were used to calculate the Cartesian coordinates of Apophis and the Apophis velocity in the barycentric equatorial system by the following algorithm (see [19,20,31,32]).

From the Kepler equation

$$
E-e \cdot \sin E=M
$$

we calculate the eccentric anomaly $E$ and, then, from $E$, the true anomaly $\varphi_{0}$ :

$$
\varphi_{0}=2 \cdot \operatorname{arctg}[\sqrt{(1+e) /(1-e)} \cdot \operatorname{tg}(0.5 \cdot E)]
$$

In subsequent calculations, we used results for the two-body interaction (the Sun and the asteroid) [21,32]. The trajectory equation of the body in a polar coordinate system with origin at the Sun has the form:

$$
r=\frac{R_{p}}{\left(\alpha_{1}+1\right) \cos \varphi-\alpha_{1}}
$$

where the polar angle $\varphi$, or, in astronomy, the true anomaly, is reckoned from the perihelion position $r=R_{p}$; $\alpha_{1}=-1 /(1+e)$ is the trajectory parameter; and $R_{p}=$ $a \cdot\left(2 a_{1}+1\right) / a_{1}$ is the perihelion radius. The expressions for the radial $v_{r}$ and transversal $v_{t}$ velocities are

$$
v_{r}= \pm v_{p} \sqrt{\left(\alpha_{1}+1\right)^{2}-\left(\alpha_{1}+1 / \bar{r}\right)^{2}}, v_{t}=v_{p} / \bar{r},(6)
$$

where for $\varphi>\pi$ we have $v_{r}<0 ; \bar{r}=r / R_{p}$ is the dimensionless radius, and the velocity at perihelion is

$$
v_{p}=\sqrt{G\left(m_{S}+m_{A s}\right) /\left(-\alpha_{1}\right) R_{p}},
$$

where $m_{S}=m_{11}$ is the Sun mass (the value of $m_{11}$ is given

\begin{tabular}{|c|c|c|c|c|}
\hline \multirow{2}{*}{$\begin{array}{c}\text { Bodies, } \\
\quad j\end{array}$} & \multicolumn{4}{|c|}{ Bodies masses in $\mathrm{kg}$, their coordinates in $\mathrm{m}$ and velocities in $\mathrm{m} \cdot \mathrm{s}^{-1}$} \\
\hline & $m_{b j}$ & $x_{a j}, v_{x a j}$ & $y_{a j}, v_{y a j}$ & $z_{a j}, v_{z a j}$ \\
\hline \multirow{2}{*}{1} & \multirow{2}{*}{$3.30187842779737 \mathrm{E}+23$} & -17405931955.9539 & -60363374194.7243 & -30439758390.4783 \\
\hline & & 37391.7107852059 & -7234.98671125365 & -7741.83625612424 \\
\hline \multirow{2}{*}{2} & \multirow{2}{*}{$4.86855338156022 \mathrm{E}+24$} & 108403264168.357 & -2376790191.8979 & -7929035215.64079 \\
\hline & & 1566.99276862423 & 31791.7241663148 & 14204.3084779893 \\
\hline \multirow{2}{*}{3} & \multirow{2}{*}{$5.97369899544255 \mathrm{E}+24$} & 55202505242.89 & 125531983622.895 & 54422116239.8628 \\
\hline & & -28122.5041342966 & 10123.4145376039 & 4387.99294255716 \\
\hline \multirow{2}{*}{4} & \multirow{2}{*}{$6.4185444055007 \mathrm{E}+23$} & -73610014623.8562 & -193252991786.298 & -86651102485.4373 \\
\hline & & 23801.7499674501 & -5108.24106287744 & -2985.97021694235 \\
\hline \multirow{2}{*}{5} & \multirow{2}{*}{$1.89900429500553 \mathrm{E}+27$} & 377656482631.376 & -609966433011.489 & -270644689692.231 \\
\hline & & 11218.8059775149 & 6590.8440254003 & 2551.89467211952 \\
\hline \multirow{2}{*}{6} & \multirow{2}{*}{$5.68604198798257 \mathrm{E}+26$} & -1350347198932.98 & 317157114908.705 & 189132963561.519 \\
\hline & & -3037.18405985381 & -8681.05223681593 & -3454.56564456648 \\
\hline \multirow{2}{*}{7} & \multirow{2}{*}{$8.68410787490547 \mathrm{E}+25$} & 2972478173505.71 & -397521136876.741 & -216133653111.407 \\
\hline & & 979.784896813787 & 5886.28982058747 & 2564.10192504801 \\
\hline \multirow{2}{*}{8} & \multirow{2}{*}{$1.02456980223201 \mathrm{E}+26$} & 3605461581823.41 & -2448747002812.46 & -1092050644334.28 \\
\hline & & 3217.00932811768 & 4100.99137103454 & 1598.60907148943 \\
\hline \multirow{2}{*}{9} & \multirow{2}{*}{$1.65085753263927 \mathrm{E}+22$} & 53511484421.7929 & -4502082550790.57 & -1421068197167.72 \\
\hline & & 5543.83894965145 & -290.586427181992 & -1757.70127979299 \\
\hline \multirow{2}{*}{10} & \multirow{2}{*}{$7.34767263035645 \mathrm{E}+22$} & 55223150629.6233 & 125168933272.726 & 54240546975.7587 \\
\hline & & -27156.1163326908 & 10140.7572420768 & 4468.97456956941 \\
\hline \multirow{2}{*}{11} & \multirow{2}{*}{$1.98891948976803 \mathrm{E}+30$} & 0 & 0 & 0 \\
\hline & & 0 & 0 & 0 \\
\hline \multirow{2}{*}{$12 \mathrm{a}$} & \multirow{2}{*}{30917984100.3039} & -133726467471.667 & -60670683449.3631 & -26002486763.62 \\
\hline & & 16908.9331065445 & -21759.6060221801 & -7660.90393288287 \\
\hline \multirow{2}{*}{$12 \mathrm{~b}$} & \multirow{2}{*}{1570796326794.9} & 314388505090.346 & 171358408804.935 & 127272183810.191 \\
\hline & & -5995.33838888362 & 9672.35319009371 & 6838.06006342785 \\
\hline
\end{tabular}
in Table 2), and $m_{A s}=m_{12}$ is the Apophis mass.

Table 2. The masses $m_{b j}$ of the planets from Mercury to Pluto, the Moon, the Sun (1 - 11) and asteroids: Apophis (12a) and $1950 \mathrm{DA}(12 \mathrm{~b})$, and the initial condition on epoch $J D_{0}=2454800.5$ (November 30, 2008) in the heliocentric equatorial coordinate system on epoch $2000.0 J D_{S}=2451545 . G=6.67259 \mathrm{E}-11 \mathrm{~m}^{3} \cdot \mathrm{s}^{-2} \cdot \mathrm{kg}^{-1}$. 
The time during which the body moves along an elliptic orbit from the point of perihelion to an orbital position with radius $\bar{r}$ is given by

$$
\begin{aligned}
& t=\frac{R_{p}}{v_{p}} \frac{\bar{r}\left|\bar{v}_{r}\right|}{2 \alpha_{1}+1} \\
& -\frac{R_{p}}{v_{p}} \frac{\alpha_{1}\left(\pi / 2+\arcsin \left\{\left[\left(2 \alpha_{1}+1\right) \bar{r}-\alpha_{1}\right] /\left(-\alpha_{1}-1\right)\right\}\right)}{\left(-2 \alpha_{1}-1\right)^{3 / 2}}
\end{aligned}
$$

where $\bar{v}_{r}=v_{r} / v_{p}$ is the dimensionless radial velocity.

At the initial time $t_{0}=0$, which corresponds to epoch $J D_{0}$ (see Table 1), the polar radius of the asteroid $r_{0}$ as dependent on the initial polar angle, or the true anomaly $\varphi_{0}$, can be calculated by Equation (5). The initial radial and initial transversal velocities as functions of $r_{0}$ can be found using Equation (6).

The Cartesian coordinates and velocities in the orbit plane of the asteroid (the axis $x_{o}$ goes through the perihelion) can be calculated by the formulas

$$
\begin{aligned}
& x_{o}=r_{0} \cdot \cos \varphi_{0} ; \quad y_{o}=r_{0} \cdot \sin \varphi_{0} \\
& v_{x o}=v_{r} \cdot \cos \varphi_{0}-v_{t} \cdot \sin \varphi_{0} \\
& v_{y o}=v_{r} \cdot \sin \varphi_{0}+v_{t} \cdot \cos \varphi_{0}
\end{aligned}
$$

The coordinates of the asteroid in the heliocentric ecliptic coordinate system can be calculated as

$$
\begin{aligned}
x_{e}= & x_{o} \cdot\left(\cos \omega_{e} \cdot \cos \Omega-\sin \omega_{e} \cdot \sin \Omega \cdot \cos i_{e}\right) \\
& -y_{o} \cdot\left(\sin \omega_{e} \cdot \cos \Omega+\cos \omega_{e} \cdot \sin \Omega \cdot \cos i_{e}\right) ; \\
y_{e} & =x_{o} \cdot\left(\cos \omega_{e} \cdot \sin \Omega-\sin \omega_{e} \cdot \cos \Omega \cdot \cos i_{e}\right) \\
& -y_{o} \cdot\left(\sin \omega_{e} \cdot \sin \Omega-\cos \omega_{e} \cdot \cos \Omega \cdot \cos i_{e}\right) ; \\
z_{e} & =x_{o} \sin \omega_{e} \cdot \sin i_{e}+y_{o} \cdot \cos \omega_{e} \cdot \sin i_{e} .
\end{aligned}
$$

The velocity components of the asteroid $v_{x e}, v_{y e}$ and $v_{z e}$ in this coordinate system can be calculated by Equations analogous to (11)-(13).

Since Equation (1) are considered in a motionless equatorial coordinate system, then elliptic coordinates (11)(13) can be transformed into equatorial ones by the Equations

$$
\begin{aligned}
& x_{a}=x_{e} ; \\
& y_{a}=y_{e} \cdot \cos \varepsilon_{0}-z_{e} \cdot \sin \varepsilon_{0} ; \\
& z_{a}=y_{e} \cdot \sin \varepsilon_{0}+z_{e} \cdot \sin \varepsilon_{0}
\end{aligned}
$$

where $\varepsilon_{0}$ is the angle between the ecliptic and the equator in epoch $J D_{S}$.

The velocity components $v_{x e}, v_{y e}$ and $v_{z e}$ can be transformed into the equatorial ones $v_{x a}, v_{y a}$ and $v_{z a}$ by Equations analogous to (14). With known heliocentric equatorial coordinates of the Solar system $n$ bodies $x_{a i}, y_{a i}$, $z_{a i} i=1,2, \cdots n$, the coordinates of Solar system barycentre, for example, along axis $x$ will be:
$X_{c}=\left(\sum_{i=1}^{n} m_{i} x_{a i}\right) / M_{S s}$, where $M_{S s}=\sum_{i=1}^{n} m_{i}$ is mass of solar system bodies.

Then barycentric equatorial coordinates $x_{i}$ of asteroid and other bodies will be

$$
x_{i}=x_{a i}-X_{c} .
$$

Other coordinates $y_{i}$ and $z_{i}$ and components of velocity $v_{x i}, v_{y i}$ and $v_{z i}$ in barycentric equatorial system of coordinates are calculated by analogous equations.

In the calculations, six orbital elements from Table 1, namely, $e, a i_{e}, \Omega, \omega_{e}$, and $M$, were used. Other orbital elements were used for testing the calculated data. The perihelion radius $R_{p}$ and the aphelion radius $R_{a}=$ $-R_{p} /\left(2 a_{1}+1\right)$ were compared to $q$ and $Q$, respectively. The orbital period was calculated by Equation (8) as twice the time of motion from perihelion to aphelion $(r=$ $R_{a}$ ). The same Equation was used to calculate the moment at which the asteroid passes the perihelion $\left(r=r_{0}\right)$. The calculated values of those quantities were compared to the values of $P$ and $t_{p}$ given in Table 1. The largest relative difference in terms of $q$ and $Q$ was within $1.9 \times$ $10^{-16}$, and in terms of $P$ and $t_{p}$, within $8 \times 10^{-9}$.

The coordinates and velocities of the planets and the Moon on epoch $J D_{0}$ were calculated by the DE406/ LE406 JPL-theory [33,34]. The masses of those bodies were modified by us [18], and the Apophis mass was calculated assuming the asteroid to be a ball of diameter $d=270 \mathrm{~m}$ and density $\rho=3000 \mathrm{~kg} / \mathrm{m}^{3}$. The masses of all bodies and the initial conditions are given in Table 2.

The starting-data preparation and testing algorithm (3)(14) was embodied as a MathCad worksheet (program AstCoor2.mcd).

\section{Apophis' Encounter with the Planets and the Moon}

In the program Galactica, a possibility to determine the minimum distance $R_{\min }$ to which the asteroid approaches a celestial body over a given interval $\Delta T$ was provided. Here, we integrated Equation (1) with the initial conditions indicated in Table 2. The integration was performed on the NKS-160 supercomputer at the Computing Center SB RAS, Novosibirsk. In the program Galactica, an extended digit length (34 decimal digits) was used, and for the time step a value $d T=10^{-5}$ year was adopted. The computations were performed over three time intervals, 0 - 100 years (Figure 5(a)), 0 - - 100 years (Figure 5(b)), and 0 - 1000 years (Figure 5(c)).

In the graphs of Figure 5 the points connected with the heavy broken line show the minimal distances $R_{\min }$ to which the asteroid approaches the bodies indicated by points embraced by the horizontal line. In other words, a point in the broken line denotes a minimal distance to 


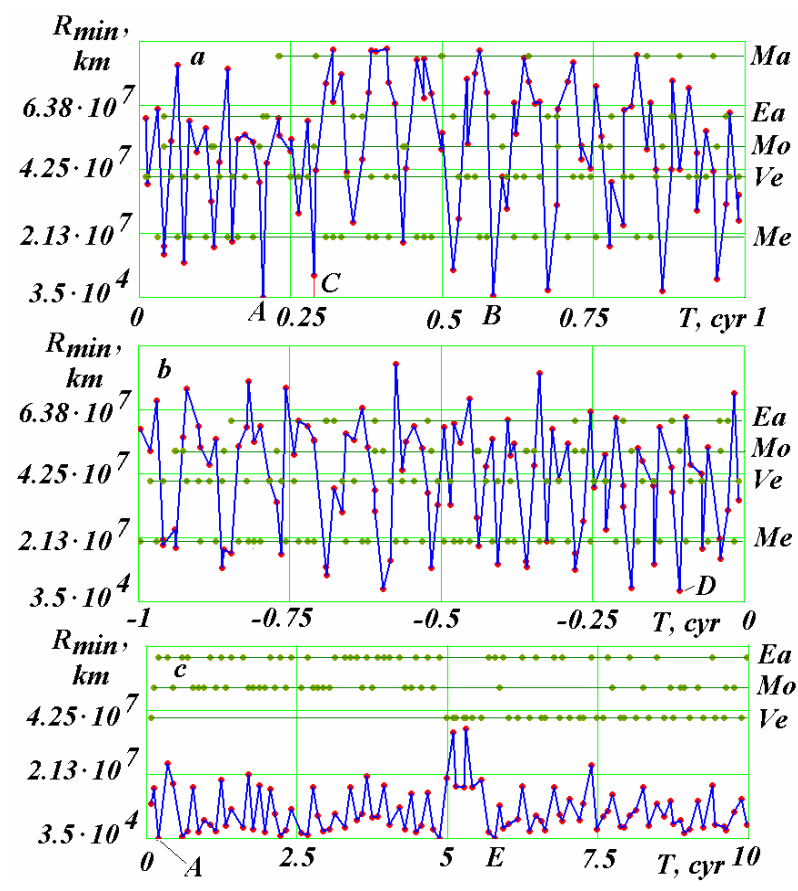

Figure 5. Apophis' encounters with celestial bodies during the time $\Delta T$ to a minimum distance $R_{\min }, \mathrm{km}$ : Mars (Ma), Earth (Ea), Moon (Mo), Venus (Ve) and Mercury (Me); a, b$\Delta T=1$ year; $\mathrm{c}-\Delta T=10$ years. $T, \operatorname{cyr}(1 \mathrm{cyr}=100 \mathrm{yr})$ is the time in Julian centuries from epoch $J D_{0}$ (November 30, 2008). Calendar dates of approach in points: $A-13$ April 2029; B-13 April 2067; C-5 September 2037; E-10 October 2586.

which, over the time $\Delta T=1$ year, the asteroid will approach a body denoted by the point in the horizontal line at the same moment. It is seen from Figure 5(a) that, starting from November 30, 2008, over the period of 100 years there will be only one Apophis' approach to the Earth (point A) at the moment $T_{\mathrm{A}}=0.203693547133403$ century to a minimum distance $R_{\operatorname{minA}}=38907 \mathrm{~km}$. A next approach (point B) will be to the Earth as well, but at the moment $T_{\mathrm{B}}=0.583679164042455$ century to a minimum distance $R_{\operatorname{minB}}=622,231 \mathrm{~km}$, which is 16 times greater than the minimum distance at the first approach. Among all the other bodies, a closest approach with be to the Moon (point D) (see Figure 5(b)) at $T_{\mathrm{D}}=$

-0.106280550824626 century to a minimum distance $R_{\operatorname{minD}}=3,545,163 \mathrm{~km}$.

In the graphs of Figures 5(a) and (b) considered above, the closest approaches of the asteroid to the bodies over time intervals $\Delta T=1$ year are shown. In integrating Equation (1) over the 1000-year interval (see Figure 5(c)), we considered the closest approaches of the asteroid to the bodies over time intervals $\Delta T=10$ years. Over those time intervals, no approaches to Mercury and Mars were identified; in other words, over the 10-year intervals the asteroid closes with other bodies. Like in Figure 5(a), there is an approach to the Earth at the moment $T_{\mathrm{A}}$. A second closest approach is also an approach to the Earth at the point $\mathrm{E}$ at $T_{\mathrm{E}}=5.778503$ century to a minimum distance $R_{\operatorname{minE}}=74002.9 \mathrm{~km}$. During the latter approach, the asteroid will pass the Earth at a minimum distance almost twice that at the moment $T_{\mathrm{A}}$.

With the aim to check the results, Equation (1) were integrated over a period of 100 years with double digit length (17 decimal digits) and the same time step, and also with extended digit length and a time step $d T=10^{-6}$ year. The integration accuracy (see Table 3) is defined [19] by the relative change of $\delta M_{\mathrm{z}}$, the $z$-projection of the angular momentum of the whole solar system for the 100 -year period. As it is seen from Table 3, the quantity $\delta M_{\mathrm{z}}$ varies from $-4.5 \times 10^{-14}$ to $1.47 \times 10^{-26}$, i.e., by 12 orders of magnitude. In the last two columns of Table 3, the difference between the moments at which the asteroid most closely approaches the Earth at point A (see Figure 5(a)) and the difference between the approach distances relative to solution 1 are indicated. In solution 2, obtained with the short digit length, the approach moment has not changed, whereas the minimum distance has reduced by $2.7 \mathrm{~m}$. In solution 3, obtained with ten times reduced integration step, the approach moment has changed by $-2 \times 10^{-6}$ year, or by -1.052 minutes. This change being smaller than the step $d T=1 \times 10^{-5}$ for solution 1 and being equal twice the step for solution 3, the value of this change provides a refinement for the approach moment. Here, the refinement for the closest approach distance by $-1.487 \mathrm{~km}$ is also obtained. On the refined calculations the Apophis approach to the Earth occurs at 21 hours 44 minutes $45 \mathrm{sec}$ on distance of $38905 \mathrm{~km}$. We emphasize here that the graphical data of Figure 5, $a$ for solutions 1 and 3 are perfectly coincident. The slight differences of solution 2 from solutions 1 and 3 are observed for $T>0.87$ century. Since all test calculations were performed considering the parameters of solution 1, it follows from here that the data that will be presented below are accurate in terms of time within 1', and in terms of distance, within $1.5 \mathrm{~km}$.

At integration on an interval of 1000 years the relative change of the angular momentum is $M_{z}=1.45 \times 10^{-20}$. How is seen from the solution 1 of Table 3 this value exceeds $M_{z}$ at integration on an interval of 100 years in 10 times, i.e. the error at extended length of number is proportional to time. It allows to estimate the error of the

Table 3. Comparison between the data on Apophis' encounter with the Earth obtained with different integration accuracies: $L_{n b}$ is the digit number in decimal digits.

\begin{tabular}{cccccc}
\hline $\begin{array}{c}\text { No. } \\
\text { solution }\end{array}$ & $L_{n b}$ & $d T, y r$ & $\delta M_{z}$ & $T_{A i}-T_{A 1}, y r$ & $\begin{array}{c}R_{\operatorname{minA} i}-R_{\operatorname{minAl}}, \\
\mathrm{km}\end{array}$ \\
\hline 1 & 34 & $1 \times 10^{-5}$ & $1.47 \times 10^{-21}$ & 0 & 0 \\
2 & 17 & $1 \times 10^{-5}$ & $-4.5 \times 10^{-14}$ & 0 & $-2.7 \times 10^{-3}$ \\
3 & 34 & $1 \times 10^{-6}$ & $1.47 \times 10^{-26}$ & $-2 \times 10^{-6}$ & -1.487 \\
\hline
\end{tabular}


second approach Apophis with the Earth in $T_{\mathrm{E}}=578$ years by results of integrations on an interval of 100 years of the solution with steps $d T=1 \times 10^{-5}$ years and $1 \times$ $10^{-6}$ years. After 88 years from beginning of integration the relative difference of distances between Apophis and Earth has become $\delta R_{88}=1 \times 10^{-4}$, that results in an error in distance of $48.7 \mathrm{~km}$ in $T_{\mathrm{E}}=578$ years.

So, during the forthcoming one-thousand-year period the asteroid Apophis will most closely approach the Earth only. This event will occur at the time $T_{\mathrm{A}}$ counted from epoch $J D_{0}$. The approach refers to the Julian day $J D_{\mathrm{A}}=2462240.406075$ and calendar date April 13, 2029, 21 hour 44'45" GMT. The asteroid will pass at a minimum distance of $38905 \mathrm{~km}$ from the Earth center, i.e., at a distance of 6.1 of Earth radii. A next approach of Apophis to the Earth will be on the 578-th year from epoch $J D_{0}$; at that time, the asteroid will pass the Earth at an almost twice greater distance.

The calculated time at which Apophis will close with the Earth, April 13, 2029, coincides with the approach times that were obtained in other reported studies. For instance, in the recent publication [4] this moment is given accurate to one minute: 21 hour 45' UTC, and the geocentric distance was reported to be in the range from 5.62 to 6.3 Earth radii, the distance of 6.1 Earth radii falling into the latter range. The good agreement between the data obtained by different methods proves the obtained data to be quite reliable.

As for the possible approach of Apophis to the Earth in 2036, there will be no such an approach (see Figure 5(a)). A time-closest Apophis' approach at the point $C$ to a minimum distance of 7.26 million $\mathrm{km}$ will be to the Moon, September 5, 2037.

\section{Apophis Orbit Evolution}

In integrating motion Equation (1) over the interval -1 century $\leq T \leq 1$ century the coordinates and velocities of the bodies after a lapse of each one year were recorded in a file, so that a total of 200 files for a one-year time interval were obtained. Then, the data contained in each file were used to integrate Equation (1) again over a time interval equal to the orbital period of Apophis and, following this, the coordinates and velocities of the asteroid, and those of Sun, were also saved in a new file. These data were used in the program DefTra to determine the parameters of Apophis' orbit relative to the Sun in the equatorial coordinate system. Such calculations were performed hands off for each of the 200 files under the control of the program PaOrb. Afterwards, the angular orbit parameters were recalculated into the ecliptic coordinate system (see Figure 6).

As it is seen from Figure 6, the eccentricity $e$ of the Apophis orbit varies non-uniformly. It shows jumps or

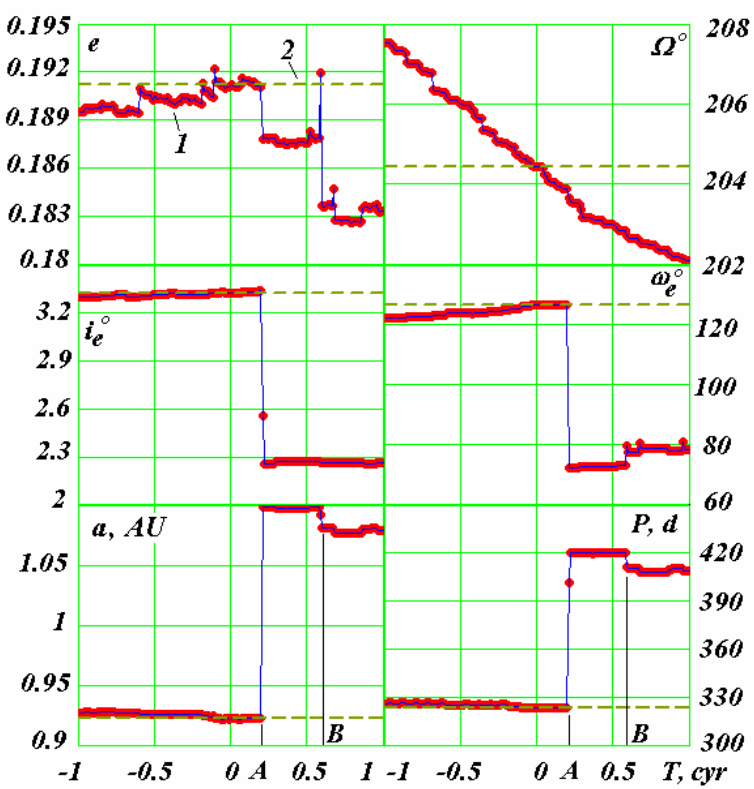

Figure 6. Evolution of Apophis' orbital parameters under the action of the planets, the Moon and the Sun over the time interval -100 years -+100 years from epoch November 30, 2008: 1-as revealed through integration of motion Equation (1); 2-initial values according to Table 1. The angular quantities: $\Omega, i_{e}$, and $\omega_{e}$ are given in degrees; the major semi-axis $a$ in $\mathrm{AU}$; and the orbital period $P$ in days.

breaks. A most pronounced break is observed at the moment $T_{\mathrm{A}}$, at which Apophis most closely approaches the Earth. A second most pronounced break is observed when Apophis approaches the Earth at the moment $T_{\mathrm{B}}$.

The longitude of ascending node $\Omega$ shows less breaks, exhibiting instead rather monotonic a decrease (see Figure 6). Other orbital elements, namely, $i_{e}, \omega_{e}$, a, and $P$, exhibit pronounced breaks at the moment of Apophis' closest pass near the Earth (at the moment $T_{\mathrm{A}}$ ).

The dashed line in Figure 6 indicates the orbit-element values at the initial time, also indicated in Table 1. As it is seen from the graphs, those values coincide with the values obtained by integration of Equation (1), the relative difference of $e, \Omega, i_{e}, \omega_{e}, a$, and $P$ from the initial values at the moment $T=0$ (see Table 1) being respectively $9.4 \times 10^{-6},-1.1 \times 10^{-6}, 3.7 \times 10^{-6},-8.5 \times 10^{-6}, 1.7 \times$ $10^{-5}$, and $3.1 \times 10^{-5}$. This coincidence testifies the reliability of computed data at all calculation stages, including the determination of initial conditions, integration of equations, determination of orbital parameters, and transformations between the different coordinate systems.

As it was mentioned in Introduction, apart from nonsimplified differential Equation (1) for the motion of celestial bodies, other equations were also used. It is a well-known fact (see Duboshin, 1976) that in perturbedmotion equations orbit-element values are used. For this reason, such equations will yield appreciable errors in determination of orbital-parameter breaks similar to 
those shown in Figure 6. Also, other solution methods for differential equations exist, including those in which expansions with respect to orbital elements or difference quotients are used. As it was already mentioned in Introduction, these methods proved to be sensitive to various resonance phenomena and sudden orbit changes observed on the approaches between bodies. Equation (1) and method (2) used in the present study are free of such shortcomings. This suggests that the results reported in the present paper will receive no notable corrections in the future.

\section{Influence of Initial Conditions}

With the purpose of check of influence of the initial conditions (IC) on Apophis trajectory the Equation (1) were else integrated on an interval 100 years with two variants of the initial conditions. The second of variant IC is given on January 04, 2010 (see Table 1). They are taken from the JPL Small-Body database [29] and correspond to the solution with number JPL sol.144, received Steven R. Chesley on October 23, 2009. In Figure 7 the results of two solutions with various IC are submitted. The line 1 shows the change in time of distance $R$ between Apophis and Earth for 100 years at the first variant IC. As it is seen from the graphs, the distance $R$ changes with oscillations, thus it is possible to determine two periods: the short period $T_{R 1}=0.87$ years and long period $T_{R 2}$. The amplitude of the short period $R_{a 1}=29.3$ million $\mathrm{km}$, and long is $R_{a 2}=117.6$ million $\mathrm{km}$. The value of the long oscillation period up to $T \sim 70$ years is equal $T_{R 20}=7.8$ years, and further it is slightly increased. After approach of April 13, 2029 (point A in Figure 7) the amplitude of the second oscillations is slightly increased. Both short and the long oscillations are not regular; therefore their average characteristics are above given.

Let's note also on the second minimal distance of Apophis approach with the Earth on interval 100 years. It occurs at the time $T_{F 1}=58.37$ years (point $F_{1}$ in Figure 7) on distance $R_{F 1}=622$ thousand $\mathrm{km}$. In April 13, 2036 (point $H$ in Figure 7) Apophis passes at the Earth on distance $R_{H 1}=86$ million $\mathrm{km}$. The above-mentioned characteristics of the solution are submitted in Table 4.

The line 2 in Figure 7 gives the solution with the second of variant IC with step of integration $d T=1 \times 10^{-5}$ years. The time of approach has coincided to within 1 minutes, and distance of approach with the second of IC became $R_{\mathrm{A} 2}=37,886 \mathrm{~km}$, i.e. has decreased on $1021 \mathrm{~km}$. To determine more accurate these parameters the Equation (1) near to point of approach were integrated with a step $d T=1 \times 10^{-6}$ years. On the refined calculations Apophis approaches with the Earth at 21 hours 44 minutes 53 second on distance $R_{\mathrm{A} 2}=37,880 \mathrm{~km}$. As it is seen from Table 4, this moment of approach differs from the moment of approach at the first of IC on 8 second. As at a

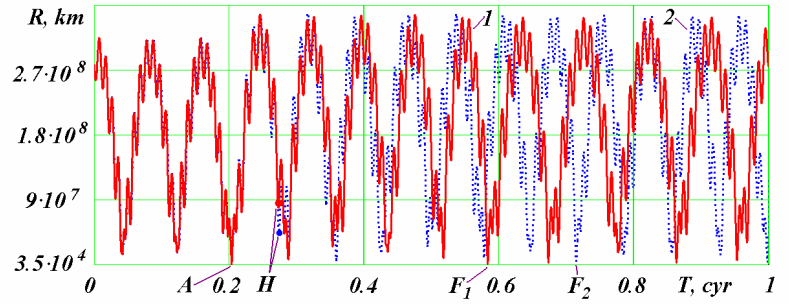

Figure 7. Evolution of distance $R$ between Apophis and Earth for 100 years. Influence of the initial conditions (IC): 1-IC from November 30, 2008; 2-IC from January 04, 2010. Calendar dates of approach in points: $A-13$ April 2029; $F_{1}-13$ April 2067; $F_{2}-14$ April 2080.

step $d T=1 \times 10^{-6}$ years the accuracy of determination of time is 16 second, it is follows, that the moments of approach coincide within the bounds of accuracy of their calculation.

The short and long oscillations at two variants IC also have coincided up to the moment of approach. After approach in point $A$ the period of long oscillations has decreased up to $T_{R 22}=7.15$ years, i.e. became less than period $T_{R 20}$ at the first variant IC. The second approach on an interval 100 years occurs at the moment $T_{F 2}=$ 70.28 years on distance $R_{F 2}=1.663$ million $\mathrm{km}$. In 2036 (point $H$ ) Apophis passes on distance $R_{H 2}=43.8$ million $\mathrm{km}$.

At the second variant of the initial conditions on January 04,2010 in comparison with the first of variant the initial conditions of Apophis and of acting bodies are changed. To reveal only errors influence of Apophis IC, the third variant of IC is given (see Table 1) as first of IC on November 30, 2008, but the Apophis IC are calculated in system Horizons according to JPL sol.144. How follows from Table 1, from six elements of an orbit $e, a$, $i_{e}, \Omega, \omega_{e}$ and $M$ the differences of three ones: $i_{e}, \Omega$ и $\omega_{e}$ from similar elements of the first variant of IC are 2.9, 1.6 and 1.5 appropriate uncertainties. The difference of other elements does not exceed their uncertainties.

At the third variant of IC with step of integration $d T=$ $1 \times 10^{-5}$ year the moment of approach has coincided with that at the first variant of IC. The distance of approach became $R_{\mathrm{A} 3}=38,814 \mathrm{~km}$, i.e. has decreased on $93 \mathrm{~km}$. For more accurate determination of these parameters the Equation (1) near to a point of approach were also integrated with a step $d T=1 \times 10^{-6}$ year. On the refined calculations at the third variant of IC Apophis approaches with the Earth at 21 hours 44 minutes 45 second on distance $R_{\mathrm{A} 3}=38,813 \mathrm{~km}$. These and other characteristics of the solution are given in Table 4. In comparison with the first variant IC it is seen, that distance of approach in 2036 and parameters of the second approach in point $F_{1}$ are slightly changed. The evolution of distance $R$ in a Figure 7 up to $T=0.6$ centuries practically coincides with the first variant (line 1). 
Table 4. Influence of the initial conditions on results of integration of the Equation (1) by program Galactica and of the equations of Apophis motion by system Horizons: Time $_{\mathrm{A}}$ and $\boldsymbol{R}_{\min }$ are time and distance of Apophis approach with the Earth in April 13, 2029, accordingly; $R_{H}$ is distance of passage Apophis with the Earth in April 13, 2036; $T_{F}$ and $R_{F}$ are time and distance of the second approach (point $F$ on Figure 7).

\begin{tabular}{|c|c|c|c|c|c|c|}
\hline \multirow{5}{*}{ Parameters } & \multicolumn{6}{|c|}{ Solutions at different variants of initial conditions } \\
\hline & \multicolumn{3}{|c|}{ Galactica } & \multicolumn{3}{|c|}{ Horizons } \\
\hline & 1 & 2 & 3 & 1 & 2 & 3 \\
\hline & 30.11 .2008 & 04.01 .2010 & 30.11 .2008 & 18.07 .2006 & 30.11 .2008 & 04.01 .2010 \\
\hline & JPL sol.140 & JPL sol.144 & JPL sol.144 & JPL sol.144 & JPL sol.140 & JPL sol.144 \\
\hline Time $_{\mathrm{A}}$ & $21: 44: 45$ & $21: 44: 53$ & $21: 44: 45$ & $21: 46: 47$ & $21: 45: 47$ & $21: 44: 45$ \\
\hline$R_{H}, 10^{6} \mathrm{~km}$ & 86.0 & 43.8 & 81.9 & 51.9 & 55.9 & 51.8 \\
\hline $\begin{array}{c}T_{F}, \text { cyr } \\
\text { from } 30.11 .08\end{array}$ & 0.5837 & 0.7138 & 0.6537 & 0.4237 & 0.9437 & 0.4238 \\
\hline$R_{F}, 10^{3} \mathrm{~km}$ & 622 & 1663 & 585 & 1515 & 684 & 1541 \\
\hline
\end{tabular}

It is seen (Table 4) that the results of the third variant differ from the first one much less than from the second variant. In the second variant the change of positions and velocities of acting bodies since November 30, 2008 for 04.01.2010 is computed under DE406, and in the third variant it does under the program Galactica. The initial conditions for Apophis in two variants are determined according to alike JPL sol.144, i.e. in these solutions the IC differ for acting bodies. As it is seen from Table 4, the moment of approach in solutions 2 and 3 differs on 8 seconds, and the approach distance differs on $933 \mathrm{~km}$. Other results of the third solution also differ in the greater degree with second ones, in comparison of the third solution with first one. It testifies that the differences IC for Apophis are less essential in comparison with differences of results of calculations under two programs: Galactica and DE406 (or Horizons).

So, the above-mentioned difference of the initial conditions (variants 1 and 3 tab. 4) do not change the time of approach of April 13, 2029, and the distance of approach in these solutions differ on $102 \mathrm{~km}$. Other characteristics: $R_{H}, T_{F}$ and $R_{F}$ also change a little. Therefore it is possible to make a conclusion, that the further refinement of Apophis IC will not essentially change its trajectory.

The same researches on influence of the initial conditions we have carried out with the integrator of NASA. In system Horizons (the JPL Horizons On-Line Ephemeris System, manual look on a site

http://ssd.jpl.nasa.gov/?horizons_doc) there is opportunity to calculate asteroid motion on the same standard dynamic model (SDM), on which the calculations in paper [4] are executed. Except considered two IC we used one more IC for Apophis at date of July 12, 2006, which is close to date of September 01, 2006 in paper [4]. The characteristics and basic results of all solutions are given in Table 4. In these solutions the similar results are received. For example, for 3-rd variant of Horizons the graphs $R$ in a Figure 7 up to $T=0.45$ centuries practically has coincided with 2-nd variant of Galactica. The time of approach in April 13, 2029 changes within the bounds of 2 minutes, and the distance is close to 38,000 $\mathrm{km}$. The distance of approach in April 13, 2036 changes from 52 up to 56 million $\mathrm{km}$. The characteristics of second approach for 100 years changes in the same bounds, as for the solutions on the program Galactica. The abovementioned other relations about IC influence have also repeated for the NASA integrator.

So, the calculations at the different initial conditions have shown that Apophis in 2029 will be approached with the Earth on distance 38 - 39 thousand $\mathrm{km}$, and in nearest 100 years it once again will approach with the Earth on distance not closer 600 thousand $\mathrm{km}$.

\section{Examination of Apophis' Trajectory in the Vicinity of Earth}

In order to examine the Apophis trajectory in the vicinity of Earth, we integrated Equation (1) over a two-year period starting from $T_{1}=0.19$ century. Following each 50 integration steps, the coordinate and velocity values of Apophis and Earth were recorded in a file.

The moment $T_{\mathrm{A}}$ at which Apophis will most closely approach the Earth falls into this two-year period. The ellipse $E_{0} E_{1}$ in Figure 8 shows the projection of the two-year Earth's trajectory onto the equatorial plane $x O y$. Along this trajectory, starting from the point $E_{0}$, the Earth will make two turns. The two-year Apophis trajectory in the same coordinates is indicated by points denoted with the letters $A p$. Starting from the point $A p_{0}$, Apophis will travel the way $A p_{0} A p_{1} A p_{\mathrm{e}} A p_{2} A p_{0} A p_{1}$ to most closely approach the Earth at the point $A p_{e}$ at the time $T_{\mathrm{A}}$. After that, the asteroid will follow another path, namely, the path $A p_{e} A p_{3} A p_{f}$.

Figure 9(a) shows the trajectory of Apophis relative to 


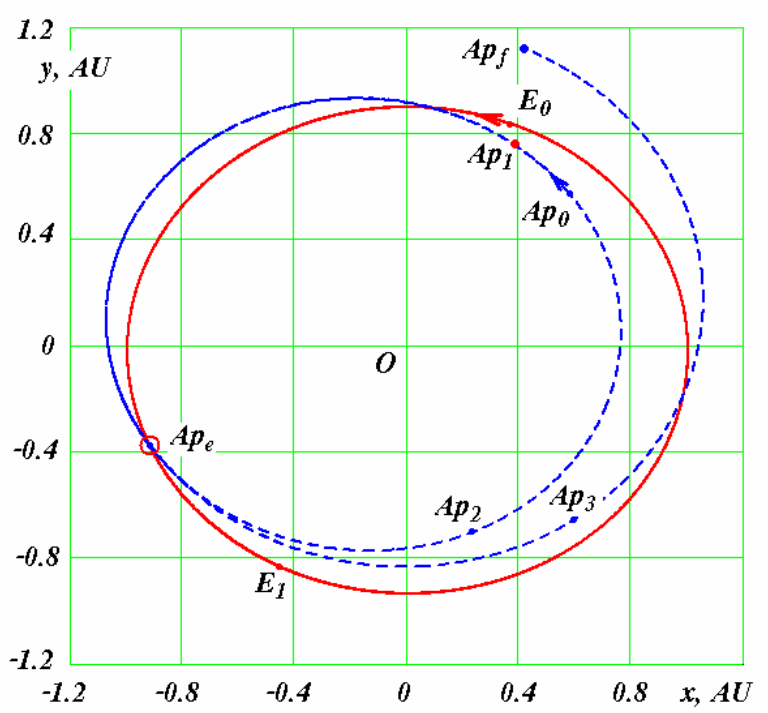

Figure 8. The trajectories of Apophis $(A p)$ and Earth $(E)$ in the barycentric equatorial coordinate system $x O y$ over a two-year period: $A p_{0}$ and $E_{0}$ are the initial position of Apophis and Earth; $A p_{f}$ is the end point of the Apophis trajectory; $A p_{e}$ is the point at which Apophis most closely approaches the Earth; the coordinates $x$ and $y$ are given in AU.

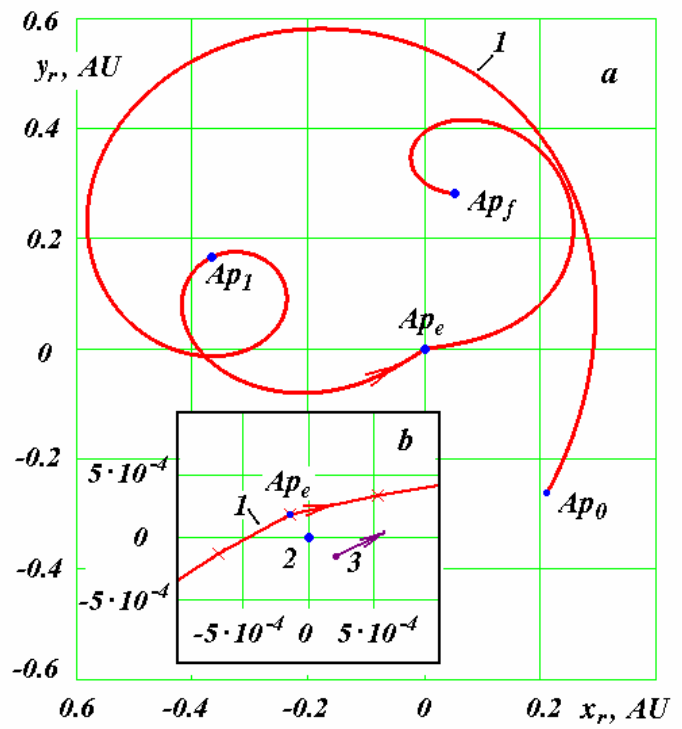

Figure 9. Apophis' trajectory (1) in the geocentric equatorial coordinate system $x_{r} O_{y_{r}}: a-0$ the normal scale, $b-0$ magnified scale on the moment of Apophis' closest approaches to the Earth (2); 3-Apophis' position at the moment of its closest approach to the Earth following the correction of its trajectory with factor $k=0.9992$ at the point $A p_{1}$; the coordinates $x_{r}$ and $y_{r}$ are given in $\mathrm{AU}$.

the Earth. Here, the relative coordinates are determined as the difference between the Apophis $(A p)$ and Earth $(E)$ coordinates:

$$
y_{r}=y_{A p}-y_{E} ; x_{r}=x_{A p}-x_{E} .
$$

Along trajectory 1 , starting from the point $A p_{0}$, Apophis will travel to the Earth-closest point $A p_{e}$, the trajectory ending at the point $A p_{f}$. The loops in the Apophis trajectory represent a reverse motion of Apophis with respect to Earth. Such loops are made by all planets when observed from the Earth (Smulsky 2007).

At the Earth-closest point $A p_{e}$ the Apophis trajectory shows a break. In Figure 9(b) this break is shown on a larger scale. Here, the Earth is located at the origin, point 2. The Sun (see Figure 8) is located in the vicinity of the barycenter $O$, i.e., in the upper right quadrant of the Earth-closest point $A p_{e}$. Hence, the Earth-closest point will be passed by Apophis as the latter will move in between the Earth and the Sun (see Figure 9(b)). As it will be shown below, this circumstance will present certain difficulties for possible use of the asteroid.

\section{Possible Use of Asteroid Apophis}

So, on April 13, 2029, we will become witnesses of a unique phenomenon, the pass of a body 31 million tons in mass near the Earth at a minimum distance of 6 Earth radii from the center of Earth. Over subsequent 1000 years, Apophis will never approach our planet closer.

Many pioneers of cosmonautics, for instance, K. E. Tsiolkovsky, Yu. A. Kondratyuk, D. V. Cole, etc. believed that the near-Earth space will be explored using large manned orbital stations. Yet, delivering heavy masses from Earth into orbit presents a difficult engineering and ecological problem. For this reason, the lucky chance to turn the asteroid Apophis into an Earth bound satellite and, then, into a habited station presents obvious interest.

Among the possible applications of a satellite, the following two will be discussed here. First, a satellite can be used to create a space lift. It is known that a space lift consists of a cable tied with one of its ends to a point at the Earth equator and, with the other end, to a massive body turning round the Earth in the equatorial plane in a 24-hour period, $P_{d}=24 \times 3600 \mathrm{sec}$. The radius of the satellite geostationary orbit is

$$
\begin{aligned}
R_{g s} & =\sqrt[3]{P_{d}^{2} G\left(m_{A}+m_{E}\right) / 4 \pi^{2}} \\
& =42241 \mathrm{~km}=6.62 R_{E e}
\end{aligned}
$$

In order to provide for a sufficient cable tension, the massive body needs to be spaced from the Earth center a distance greater than $R_{g s}$. The cable, or several such cables, can be used to convey various goods into space while other goods can be transported back to the Earth out of space.

If the mankind will become able to make Apophis an Earth bound satellite and, then, deflect the Apophis orbit into the equatorial plane, then the new satellite would suit the purpose of creating a space lift.

A second application of an asteroid implies its use as a 
"shuttle" for transporting goods to the Moon. Here, the asteroid is to have an elongated orbit with a perihelion radius close to that of a geostationary orbit and an apogee radius approaching the perigee radius of the lunar orbit. In the latter case, at the geostationary-orbit perigee goods would be transferred onto the satellite Apophis and then, at the apogee, those goods would arrive at the Moon.

The two applications will entail the necessity of solving many difficult problems which now can seem even unsolvable. On the other hand, none of those problems will be solved at all without making asteroid an Earth satellite. Consider now the possibilities available here.

The velocity of the asteroid relative to the Earth at the Earth-closest point $A p_{e}$ is $v_{A E}=7.39 \mathrm{~km} \cdot \mathrm{s}^{-1}$. The velocity of an Earth bound satellite orbiting at a fixed distance $R_{\operatorname{minA}}$ from the Earth (circular orbit) is

$$
v_{C E}=\sqrt{G\left(m_{A}+m_{E}\right) / R_{\min \mathrm{A}}}=3.2 \mathrm{~km} \cdot \mathrm{s}^{-1}
$$

For the asteroid to be made an Earth-bound satellite, its velocity $v_{A E}$ should be brought close to $v_{C E}$. We performed integration of Equation (1) assuming the Apophis velocity at the moment $T_{A}$ to be reduced by a factor of 1.9, i.e., the velocity $v_{A E}=7.39 \mathrm{~km} \cdot \mathrm{s}^{-1}$ at the moment $T_{A}$ was decreased to $3.89 \mathrm{~km} \cdot \mathrm{s}^{-1}$. In the later case, Apophis becomes an Earth bound satellite with the following orbit characteristics: eccentricity $e_{s 1}=0.476$, equator-plane inclination angle $i_{s 1}=39.2^{\circ}$, major semiaxis $a_{s 1}=74540 \mathrm{~km}$, and sidereal orbital period $P_{s 1}=$ 2.344 days.

We examined the path evolution of the satellite for a period of 100 years. In spite of more pronounced oscillations of the orbital elements of the satellite in comparison with those of planetary orbit elements, the satellite's major semi-axis and orbital period proved to fall close to the indicated values. For the relative variations of the two quantities, the following estimates were obtained: $|\delta a|<$ $\pm 2.75 \times 10^{-4}$ and $|\delta P|< \pm 4.46 \times 10^{-4}$. Yet, the satellite orbits in a direction opposite both to the Earth rotation direction and the direction of Moon's orbital motion. That is why the two discussed applications of such a satellite turn to be impossible.

Thus, the satellite has to orbit in the same direction in which the Earth rotates. Provided that Apophis (see Figure 9(b)) will round the Earth from the night-side (see point 3) and not from the day-side (see line 1), then, on a decrease of its velocity the satellite will be made a satellite orbiting in the required direction.

For this matter to be clarified, we have integrated Equation (1) assuming different values of the asteroid velocity at the point $A p_{1}$ (see Figure 9). This point, located at half the turn from the Earth-closest point $A p_{e}$, will be passed by Apophis at the time $T_{A p 1}=0.149263369488169$ century. At the point $A p_{1}$ the projections of the Apophis velocity in the barycentric equatorial coordinate system are $v_{A p 1 x}=-25.6136689 \mathrm{~km} \cdot \mathrm{s}^{-1}, v_{A p 1 y}=17.75185451$ $\mathrm{km} \cdot \mathrm{s}^{-1}$, and $v_{A p 1 z}=5.95159206 \mathrm{~km} \cdot \mathrm{s}^{-1}$. In the numerical experiments, the component values of the satellite velocity were varied to one and the same proportion by multiplying all them by a single factor $k$, and then Equation (1) were integrated to determine the trajectory of the asteroid. Figure 10 shows the minimum distance to which Apophis will approach the Earth versus the value of $k$ by which the satellite velocity at the point $A p_{1}$ was reduced.

We found that, on decreasing the value of $k$ (see Figure 10), the asteroid will more closely approach the Earth, and at $k=0.9999564$ Apophis will collide with the Earth. On further decrease of asteroid velocity the asteroid will close with the Earth on the Sun-opposite side, and at $k=0.9992$ the asteroid will approach the Earth center (point 3 in Figure 9(b)) to a minimum distance $R_{\min 3}=39,157 \mathrm{~km}$ at the time $T_{3}=0.2036882$ century. This distance $R_{\min 3}$ roughly equals the distance $R_{\min }$ to which the asteroid was found to approach the Earth center while moving in between the Earth and the Sun.

In this case, the asteroid velocity relative to the Earth is also $v_{A E}=7.39 \mathrm{~km} \cdot \mathrm{s}^{-1}$. On further decrease of this velocity by a factor of 1.9 , i.e., down to $3.89 \mathrm{~km} \cdot \mathrm{s}^{-1}$ Apophis will become an Earth bound satellite with the following orbit parameters: eccentricity $e_{s 2}=0.486$, equator plane inclination angle $i_{s 2}=36^{\circ}$, major semi-axis $a_{s 2}=$ $76,480 \mathrm{~km}$, and sidereal period $P_{s 2}=2.436$ day. In addition, we investigated into the path evolution of the Earth bound satellite over a 100-year period. The orbit of the satellite proved to be stable, the satellite orbiting in the same direction as the Moon does.

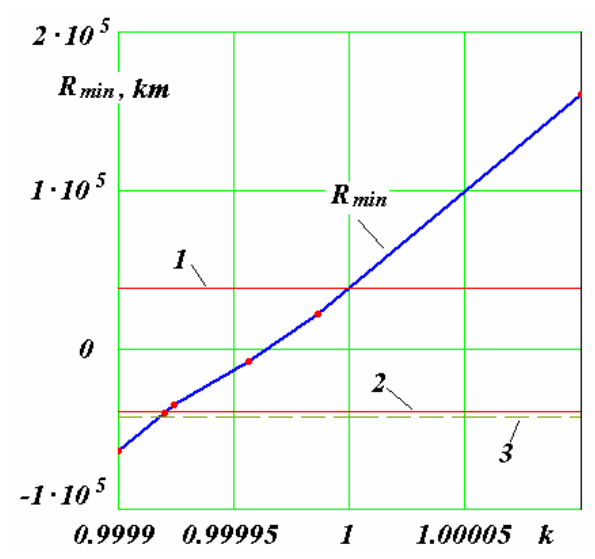

Figure 10. The minimum distance $R_{\min }$ to which Apophis will approach the Earth center versus the value of $k(k$ is the velocity reduction factor at the point $A_{p 1}$ (see Figure 8)). The positive values of $\boldsymbol{R}_{\text {min }}$ refer to the day-side: The values of $\boldsymbol{R}_{\min }$ are given in $\mathrm{km}$; 1 - the minimum distance to which Apophis will approach the Earth center on April 13, 2029 (day-side); 2 - the minimum distance to which Apophis will approach the Earth center after the orbit correction (nightside); 3 - geostationary orbit radius $\boldsymbol{R}_{g s}$. 
Thus, for Apophis to be made a near-Earth satellite orbiting in the required direction, two decelerations of its velocity need to be implemented. The first deceleration is to be effected prior to the Apophis approach to the Earth, for instance, at the point $A p_{1}$ (see Figure 8), 0.443 year before the Apophis approach to the Earth. Here, the Apophis velocity needs to be decreased by $2.54 \mathrm{~m} / \mathrm{s}$. A second deceleration is to be effected at the moment the asteroid closes with the Earth. In the case under consideration, in which the asteroid moves in an elliptic orbit, the asteroid velocity needs to be decreased by $3.5 \mathrm{~km} \cdot \mathrm{s}^{-1}$.

Slowing down a body weighing 30 million tons by 3.5 $\mathrm{km} \cdot \mathrm{s}^{-1}$ is presently a difficult scientific and engineering problem. For instance, in paper [7] imparting Apophis with a velocity of $10^{-6} \mathrm{~m} / \mathrm{s}$ was believed to be a problem solvable with presently available engineering means. On the other hand, Rykhlova et al. [7] consider increasing the velocity of such a body by about $1-2 \mathrm{~cm} / \mathrm{s}$ a difficult problem. Yet, with Apophis being on its way to the Earth, we still have a twenty-year leeway. After the World War II, even more difficult a problem, that on injection of the first artificial satellite in near-Earth orbit and, later, the launch of manned space vehicles, was successfully solved in a period of ten years. That is why we believe that, with consolidated efforts of mankind, the objective under discussion will definitely be achieved.

It should be emphasized that the authors of Giorgini et al. 2008 considered the possibility of modifying the Apophis orbit for organizing its impact onto asteroid (144898) 2004 VD17. There exists a small probability of the asteroid's impact onto the Earth in 2102. Yet, the problem on reaching a required degree of coordination between the motions of the two satellites presently seems to be hardly solvable. This and some other examples show that many workers share an opinion that substantial actions on the asteroid are necessary for making the solution of the various space tasks a realistic program.

\section{Asteroid 1950 DA Approaches to the Earth}

The distances to which the asteroid 1950 DA will approach solar-system bodies are shown versus time in Figure 11. It is seen from Figure 11(a), that, following November 30, 2008, during the subsequent 100-year period the asteroid will most closely approach the Moon: at the point $\mathrm{A}\left(T_{\mathrm{A}}=0.232532 \mathrm{cyr}\right.$ and $R_{\min }=11.09$ million $\mathrm{km})$ and at the point $\mathrm{B}\left(T_{\mathrm{B}}=0.962689 \mathrm{cyr}\right.$ and $R_{\min }=$ 5.42 million $\mathrm{km}$ ). The encounters with solar-system bodies the asteroid had over the period of 100 past years are shown in Figure 11(b). The asteroid most closely approached the Earth twice: at the point $\mathrm{C}\left(T_{\mathrm{C}}=-0.077395\right.$ cyr and $R_{\min }=7.79$ million $\left.\mathrm{km}\right)$, and at the point $\mathrm{D}\left(T_{\mathrm{D}}=\right.$ $-0.58716 \mathrm{cyr}$ and $R_{\min }=8.87$ million $\mathrm{km}$ ).

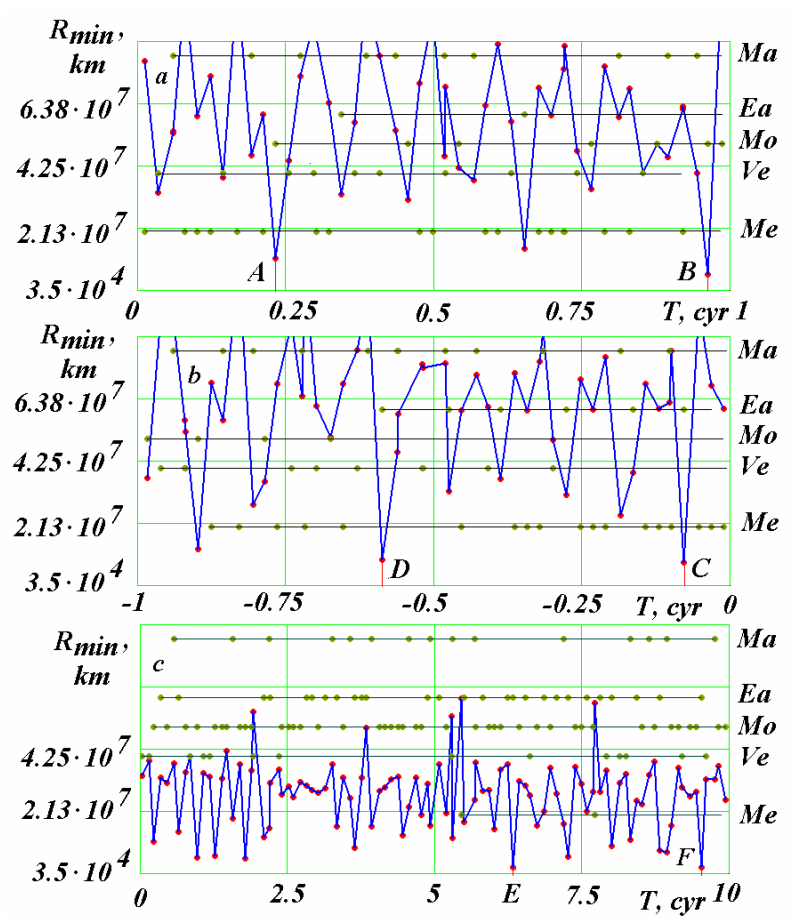

Figure 11. Approach of the asteroid 1950 DA to solar-system bodies. The approach distances are calculated with time interval $\Delta T: a, b-\Delta T=1$ year; $c-\Delta T=10$ years. $R_{\min }$, $\mathbf{k m}$ is the closest approach distance. Calendar dates of approach in points see Table 5. For other designations, see Figure 5.

Over the interval of forthcoming 1000 years, the minimal distances to which the asteroid will approach solar-system bodies on time span $\Delta T=10$ years are indicated in Figure 11(c). The closest approach of 1950 DA will be to the Earth: at the point $E\left(T_{E}=6.322500 \mathrm{cyr}\right.$ and $R_{\min }=2.254$ million $\left.\mathrm{km}\right)$, and at the point $F\left(T_{F}=\right.$ $9.532484 \mathrm{cyr}$ and $R_{\min }=2.248$ million $\mathrm{km}$ ).

To summarize, over the 1000-year time interval the asteroid 1950 DA will most closely approach the Earth twice, at the times $T_{E}$ and $T_{F}$, to a minimum distance of 2.25 million $\mathrm{km}$ in both cases. The time $T_{E}$ refers to the date March 6, 2641, and the time $T_{F}$, to the date March 7, 2962.

Giorgini et al. [35] calculated the nominal 1950 DA trajectory using earlier estimates for the orbit-element values of the asteroid, namely, the values by the epoch of March 10, 2001 (JPL sol.37). In paper [35], as the variation of initial conditions for the asteroid, ranges were set three times wider than the uncertainty in element values. For the extreme points of the adopted ranges, in the calculations 33 collision events were registered. In this connection, Giorgini et al. 2002 have entitled their publication "Asteroid 1950 DA Encounter with Earth in 2880".

We made our calculations using the orbit-element va- 
lues of 1950 DA by the epoch of November 30, 2008 (JPL sol.51) (see Table 1). By system Horizons the JPL sol.37 can be prolonged till November 30,2008. As it is seen in this case, the difference of orbital elements of the solution 37 from the solution 51 on two - three order is less, than uncertainties of orbit elements, i.e. the orbital elements practically coincide.

With the aim to trace how the difference methods of calculation has affected the 1950 DA motion, in Table 5 we give a comparison of the approach times of Figure 11 with the time-closest approaches predicted in paper [35]. According to Table 5, the shorter the separation between the approach times (see points $C$ and $A$ ) and the start time of calculation (2008-11-30), the better is the coincidence in terms of approach dates and minimal approach distances $R_{\min }$. For more remote times (see points $D$ and $B$ ) the approach times differ already by 1 day. At the point $E$, remote from the start time of calculation by 680 year, the approach times differ already by eight days, the approach distances still differing little. At the most remote point $F$, according to our calculations, the asteroid will approach the Earth in 2962 to a distance of $0.015 \mathrm{AU}$, whereas, according to the data of Giorgini et al. [35], a most close approach to the Earth, to a shorter distance, will be in 2880.

So, our calculations show that the asteroid 1950 DA will not closely approach the Earth. It should be noted that our calculation algorithm for predicting the motion of the asteroid differs substantially from that of Giorgini et al. [35]. We solve non-simplified Equation (1) by a high-precision numerical method. In doing so, we take into account the Newtonian gravitational interaction only. In paper [35], additional weak actions on the asteroid were taken into account. Yet, the position of celestial bodies acting on the asteroid is calculated from the ephemerides of DE-series. Those ephemeredes approximate observational data and, hence, they describe those data to good precision. Yet, the extent to which the pre- dicted motion of celestial bodies deviates from the actual motion of these bodies is the greater the farther the moment of interest is remote from the time interval during which the observations were made. We therefore believe that the difference between the present calculation data for the times 600 and 900 years (points $E$ and $F$ in Table 5) and the data of Giorgini et al. [35] results from the indicated circumstance.

\section{Evolution of the 1950 DA Orbit}

Figure 12 shows the evolution of 1950 DA orbital elements over a 1000 -year time interval as revealed in calculations made with time span $\Delta T=10$ years. With the passage of time, the orbit eccentricity $e$ non-monotonically increases. The angle of longitude of ascending node $\Omega$, the angle of inclination $i_{e}$ to the ecliptic plane, and the angle of perihelion argument $\omega_{e}$ show more monotonic variations. The semi-axis $a$ and the orbital period $P$ both oscillate about some mean values. As it is seen from Figure 12, at the moments of encounter with the Earth, $T_{E}$ and $T_{F}$, the semi-axis $a$ and the period $P$ show jumps. At the same moments, all the other orbit elements exhibit less pronounced jumps.

The dashed line in Figure 12 indicates the initial-time values of orbital elements presented in Table 1. As it is seen from the graphs, these values are perfectly coincident with the values for $T=0$ obtained by integration of Equation (1). The relative differences between the values of $e, \Omega, i_{e}, \omega_{e}, a$, and $P$ and the initial values of these parameters given in Table 1 are $-3.1 \times 10^{-4},-1.6 \times 10^{-5}$, $-6.2 \times 10^{-5},-1.5 \times 10^{-5},-1.5 \times 10^{-5},-1.0 \times 10^{-4}$, and $-3.0 \times 10^{-4}$, respectively. Such a coincidence validates the calculations at all stages, including the determination of initial conditions, integration of Equation (1), determination of orbital-parameter values, and the transformation between different coordinate systems.

It should be noted that the relative difference for the

Table 5. Comparison between the data on asteroid 1950 DA encounters with the Earth and Moon: Our data are denoted with characters $A, B, C, D, E, F$, as in Figure 11, and the data by Giorgini et al. [35] are denoted as Giorg.

\begin{tabular}{|c|c|c|c|c|c|}
\hline Source & $J D$, days & Date & Time, days & Body & $R_{\min }, \mathrm{AU}$ \\
\hline$D$ & 2433354 & $1950-03-13$ & 0.730 & Earth & 0.059273 \\
\hline Giorg. & - & $1950-03-12$ & 0.983 & Earth & 0.059286 \\
\hline$C$ & 2451973 & $2001-03-05$ & 0.157 & Earth & 0.052075 \\
\hline Giorg. & - & 2001-03-05 & 0.058 & Earth & 0.052073 \\
\hline$A$ & 2463293 & $2032-03-02$ & 0.222 & Moon & 0.074158 \\
\hline Giorg. & - & $2032-03-02$ & 0.281 & Earth & 0.075751 \\
\hline$B$ & 2489962 & 2105-03-09 & 0.224 & Moon & 0.036260 \\
\hline Giorg. & - & $2105-03-10$ & 0.069 & Earth & 0.036316 \\
\hline$E$ & 2685729 & $2641-03-06$ & 0.338 & Earth & 0.015070 \\
\hline Giorg. & - & $2641-03-14$ & 0.330 & Earth & 0.015634 \\
\hline$F$ & 2802974 & $2962-03-07$ & 0.985 & Earth & 0.015030 \\
\hline Giorg. & - & $2880-03-16$ & 0.836 & Earth & 0.001954 \\
\hline
\end{tabular}




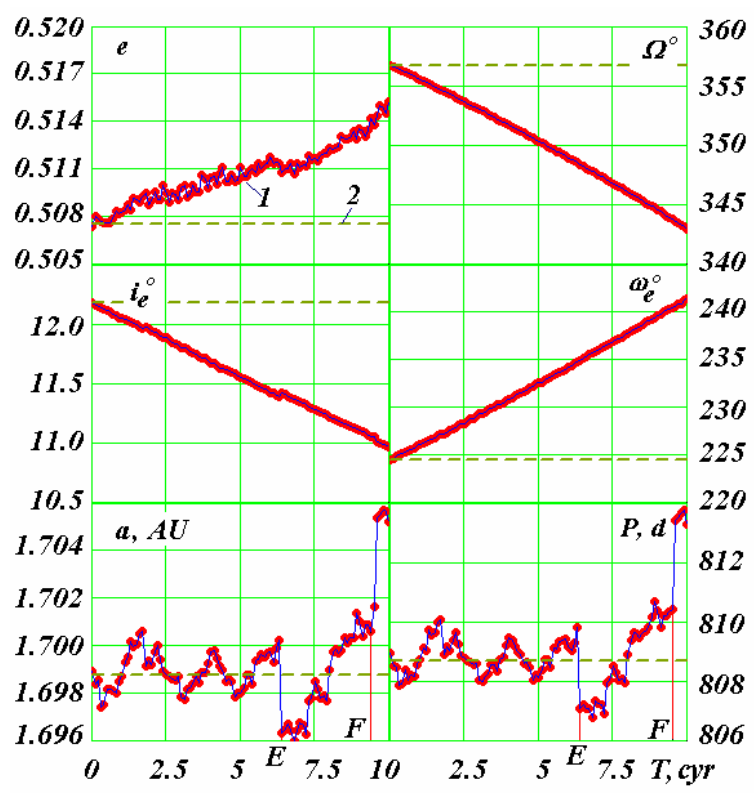

Figure 12. Evolution of 1950 DA orbital parameters under the action of the planets, the Moon, and the Sun over the time interval 0 - 1000 from the epoch November 30, 2008: 1-As revealed through integration of motion Equation (1) obtained with the time interval $\Delta T=10$ years: 2-Initial values according to Table 1 . The angular quantities, $\Omega, i_{e}$, and $\omega_{e}$, are given in degrees, the major semi-axis $a-$ in $A U$, and the orbital period $P$, in days.

same elements of Apophis is one order of magnitude smaller. The cause for the latter can be explained as follows. Using the data obtained by integrating Equation (1), we determine the orbit elements at the time equal to half the orbital period. Hence, our elements are remote from the time of determination of the initial conditions by that time interval. Since the orbital period of Apophis is shorter than that of $1950 \mathrm{DA}$, the time of determination of Apophis' elements is 0.66 year closer in time to the time of determination of initial conditions than the same time for 1950 DA.

\section{Study of the $1950 \mathrm{DA}$ Trajectory in the Encounter Epoch of March 6, 2641}

Since the distances to which the asteroid will approach the Earth at the times $T_{E}$ and $T_{F}$ differ little, consider the trajectories of the asteroid and the Earth at the nearest approach time $T_{E}$, March 6, 2641. The ellipse $E_{0} E_{f}$ in Figure 13 shows the projection of the Earth trajectory over a 2.5-year period onto the equatorial plane $x O y$. This projection shows that, moving from the point $E_{0}$ the Earth will make 2.5 orbital turns. The trajectory of 1950 DA starts at the point $A_{0}$. At the point $A_{e}$ the asteroid will approach the Earth in 2641 to a distance of $0.01507 \mathrm{AU}$.

The post-encounter trajectory of the asteroid remains roughly unchanged. Then, the asteroid will pass through

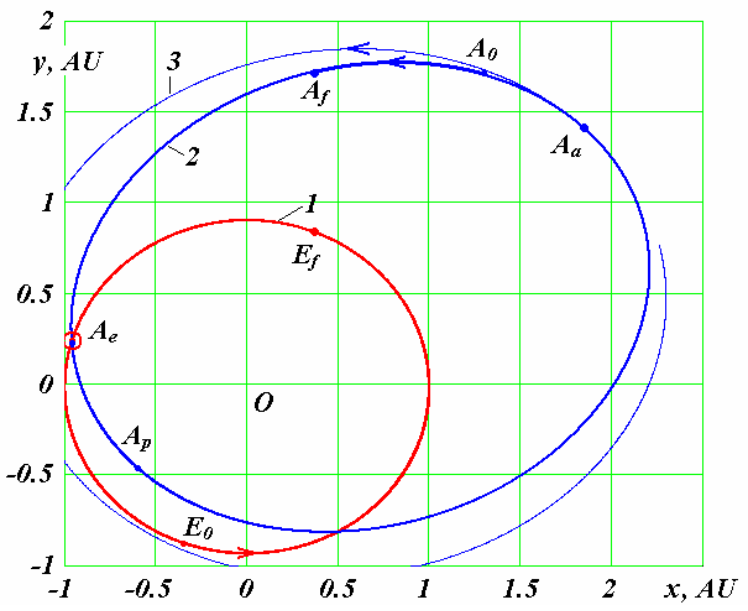

Figure 13. The trajectories of Earth (1) and 1950 DA (2) in the barycentric equatorial coordinate system $x O y$ over 2.5 years in the encounter epoch of March 6, 2641 (point $A_{e}$ ): $A_{0}$ and $E_{0}$ are the starting points of the $1950 \mathrm{DA}$ and Earth trajectories; $A_{f}$ and $E_{f}$ are the end points of the $1950 \mathrm{DA}$ and Earth trajectories; 3-1950 DA trajectory after the correction applied at the point $A_{a}$ is shown arbitrarily; the coordinates $x$ and $y$ are given in AU.

the perihelion point $A_{p}$ and aphelion point $A_{a}$, and the trajectory finally ends at the point $A_{f}$.

Figure 14(a) shows the trajectory of the asteroid relative to the Earth. The relative coordinates $x_{r}$ and $y_{r}$ were calculated by a Equation analogous to (15). Starting at the point $A_{0}$, the asteroid $1950 \mathrm{DA}$ will move to the point $A_{e}$, where it will most closely approach the Earth, the end point of the trajectory being the point $A_{f}$. The loop in the 1950 DA trajectory represents a reverse motion of the asteroid relative to the Earth.

On an enlarged scale, the encounter of the asteroid with the Earth is illustrated by Figure 14(b). The Sun is in the right upper quadrant. The velocity of the asteroid relative to the Earth at the closing point $A_{e}$ is $v_{A E}=14.3$ $\mathrm{km} \cdot \mathrm{s}^{-1}$.

\section{Making the Asteroid 1950 DA an Earth-Bound Satellite}

Following a deceleration at the point $A_{e}$ (see Figure 14(b)), the asteroid 1950 DA can become a satellite orbiting around the Earth in the same direction as the Moon does. At this point $E$ (see Table 5) the distance from the asteroid to the Earth's center is $R_{\min E}=2.25$ million km, the mass of the asteroid being $m_{A}=1.57$ milliard ton. According to (17), the velocity of a satellite moving in a circular orbit of radius $R_{\min E}$ is $v_{C E}=0.421 \mathrm{~km} \cdot \mathrm{s}^{-1}$. For the asteroid 1950 DA to be made a satellite, its velocity needs to be brought close to the value $v_{C E}$ or, in other words, the velocity of the asteroid has to be decreased by $\Delta V \approx 13.9 \mathrm{~km} \cdot \mathrm{s}^{-1}$. In this situation, the asteroid's momentum will become decreased by a value $m_{a} \Delta V=$ 


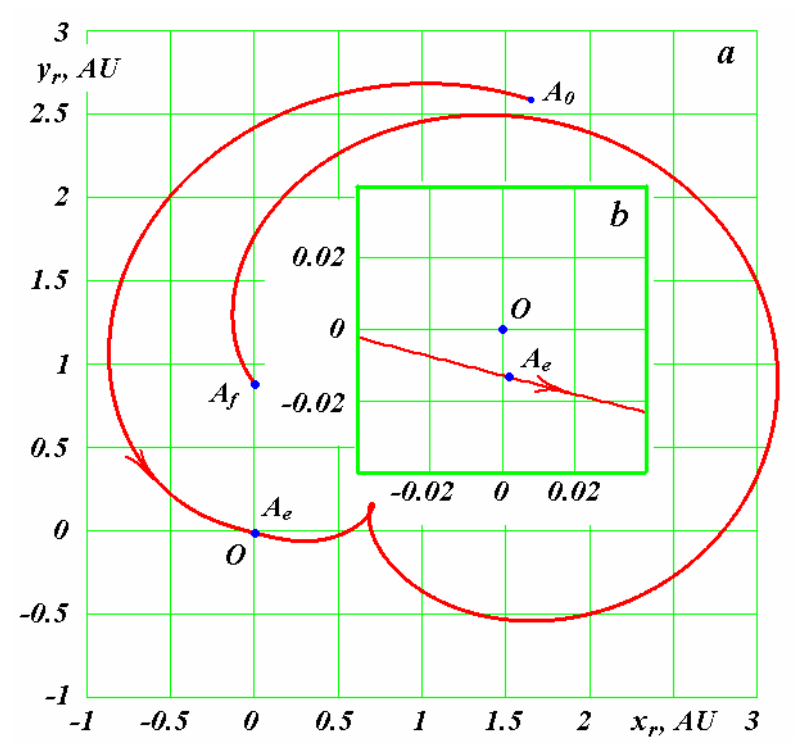

Figure 14. The 1950 DA trajectory in the geocentric equatorial coordinate system $x_{r} O y_{r}: a-O n$ ordinary scale; $b-O n$ an enlarged scale by the moment of 1950 DA encounter with the Earth: point $O$-The Earth, point $A_{\mathrm{e}}$-The asteroid at the moment of its closest approach to the Earth; the coordinates $x_{r}$ and $y_{r}$ are given in $\mathrm{AU}$.

$2.18 \times 10^{16} \mathrm{~kg} \cdot \mathrm{m} / \mathrm{s}$, for Apophis the same decrease amounts to $m_{a} \cdot \Delta V=1.08 \times 10^{14} \mathrm{~kg} \cdot \mathrm{m} \cdot \mathrm{s}^{-1}$, a 200 times greater value. Very probably, satellites with an orbital radius of 2.25 million $\mathrm{km}$ will not find a wide use. In this connection, consider another strategy for making the asteroid an Earth-bound satellite. Suppose that the velocity of the asteroid at the aphelion of its orbit (point $A_{a}$ in Figure 13) was increased so that the asteroid at the orbit perihelion has rounded the Earth orbit on the outside of it passing by the orbit at a distance $R_{1}$. To simplify calculations, we assume the Earth's orbit to be a circular one with a radius equals the semi-axis of the Earth orbit $a_{E}=1 \mathrm{AU}$. So, in the corrected orbit of the asteroid the perihelion radius will be

$$
R_{p c}=a_{E}+R_{1}
$$

Then, let us decrease the velocity of the asteroid at the perihelion of the corrected orbit to a value such that to make the asteroid an Earth-bound satellite. To check efficiency of this strategy, perform required calculations based on the two-body interaction model for the asteroid and the Sun (Smulsky 2007, Smulsky 2008). We write the expression for the parameter of trajectory in three forms:

$$
\alpha_{1}=-0.5\left(1+R_{p} / R_{a}\right)=\frac{\mu_{1}}{R_{p} \cdot v_{p}^{2}}=\frac{R_{p} \mu_{1}}{R_{a}^{2} \cdot v_{a}^{2}},
$$

where

$$
\mu_{1}=-G\left(m_{s}+m_{A s}\right)
$$

is the interaction parameter of the Sun and the asteroid, $m_{s}$ is the Sun mass, $m_{A s}$ is the asteroid mass, and $\alpha_{1}=$ -0.6625 is the $1950 \mathrm{DA}$ trajectory parameter.

Then, using (19), for the corrected orbit of the asteroid with parameters $R_{p c}$ and $v_{a c}$ we obtain:

$$
-0.5\left(1+R_{p c} / R_{a}\right)=\frac{R_{p c} \mu_{1}}{R_{a}^{2} v_{a c}^{2}}
$$

From (21), we obtain the corrected velocity of the asteroid at aphelion:

$$
v_{a c}=\sqrt{\frac{2 \cdot R_{p c}\left(-\mu_{1}\right)}{R_{a}^{2}\left(R_{a}+R_{p c}\right)}}
$$

Using (19), we express $\mu_{1}$ in terms of $\alpha_{1}$ and $v_{a}$, and after substitution of this expression into (22) we obtain the corrected velocity at aphelion:

$$
v_{a c}=v_{a} \sqrt{\frac{2\left(-\alpha_{1}\right) R_{p c} \cdot R_{a}}{\left(R_{a}+R_{p c}\right) \cdot R_{p}}} .
$$

From the second Kepler law, $R_{a} \cdot v_{a c}=R_{p c} \cdot v_{p c}$, we determine the velocity at the perihelion of the corrected orbit:

$$
v_{p c}=v_{a c} \cdot R_{a} / R_{p c} .
$$

As a numerical example, consider the problem on making the asteroid 1950 DA an Earth-bound satellite with a perihelion radius equal to the geostationary orbit radius $R_{1}=R_{g s}=42,241 \mathrm{~km}$. Prior to the correction, the aphelion velocity of the asteroid is $v_{a}=13.001 \mathrm{~km} \cdot \mathrm{s}^{-1}$, whereas the post-correction velocity calculated by Equation (23) is $v_{a c}=13.912 \mathrm{~km} \cdot \mathrm{s}^{-1}$. Thus, for making the asteroid a body rounding the Earth orbit it is required to increase its velocity at the point $A_{a}$ in Figure 13 by 0.911 $\mathrm{km} \cdot \mathrm{s}^{-1}$. The corrected orbit is shown in Figure 13 with line 3 .

According to (24), the velocity of the asteroid at the perihelion of the corrected orbit is $v_{p c}=35.622 \mathrm{~km} \cdot \mathrm{s}^{-1}$. Using Equation (7), for a circular Earth orbit with $\alpha_{1}=$ -1 and $R_{p}=a_{E}$, and with the asteroid mass $m_{A S}$ replaced with the Earth mass $m_{E}$, for the orbital velocity of the Earth we obtain a value $v_{O E}=29.785 \mathrm{~km} \cdot \mathrm{s}^{-1}$. According to (17), the velocity of the satellite in the geostationary orbit is $v_{g s}=3.072 \mathrm{~km} \cdot \mathrm{s}^{-1}$. Since those velocities add up, for the asteroid to be made an Earth satellite, its velocity has to be decreased to the value $v_{O E}+v_{C E}=32.857$ $\mathrm{km} \cdot \mathrm{s}^{-1}$. Thus, the asteroid 1950 DA will become a geostationary satellite following a decrease of its velocity at the perihelion of the corrected orbit by $v_{p c}-\left(v_{O E}+v_{C E}\right)=$ $2.765 \mathrm{~km} \cdot \mathrm{s}^{-1}$.

We have performed the calculations for the epoch of 2641. Those calculations are, however, valid for any epoch. Our only concern is to choose the time of 1950 DA orbit 
correction such that at the perihelion of the corrected orbit the asteroid would approach the Earth. Such a problem was previously considered in Smulsky 2008, where a launch time of a space vehicle intended to pass near the Venus was calculated. The calculations by Equations (18)-(24) were carried out on the assumption that the orbit planes of the asteroid and the Earth, and the Earth equator plane, are coincident. The calculation method of Smulsky 2008 allows the calculations to be performed at an arbitrary orientation of the planes. In the same publication it was shown that, following the determination of the nearest time suitable for correction, such moments in subsequent epochs can also be calculated. They follow at a certain period.

In the latter strategy for making the asteroid $1950 \mathrm{DA}$ a near-Earth satellite, a total momentum $m_{a} \cdot \Delta V=$ $m_{a} \cdot(0.911+2.765) \times 10^{3}=5.77 \times 10^{15} \mathrm{~kg} \cdot \mathrm{m} / \mathrm{s}$ needs to be applied. This value is 4.8 times smaller than that in the former strategy and 53 times greater than the momentum required for making Apophis an Earth satellite. It seems more appropriate to start the creation of such Earth satellites with Apophis. In book [36], page 189, it is reported that an American astronaut Dandridge Cole and his co-author Cox [37] advanced a proposal to capture planetoids in between the Mars and Jupiter and bring them close to the Earth. Following this, mankind will be able to excavate rock from the interior of the planetoids and, in this way, produce in the cavities thus formed artificial conditions suitable for habitation. Note that another possible use of such satellites mentioned in [37] is the use of ores taken from them at the Earth.

Although the problem on making an asteroid an Earth satellite is a problem much easier to solve than the problem on planetoid capture, this former problem is nonetheless also a problem unprecedented in its difficulty. Yet, with this problem solved, our potential in preventing the serious asteroid danger will become many times enhanced. That is why, mankind getting down to tackling the problem, this will show that we have definitely passed from pure theoretical speculations in this field to practical activities on Earth protection of the asteroid hazard.

\section{Discussion}

In the 20th century, in the science of motion, namely, in the mechanics the changes have been introduced that led to the opinion of the movements' indeterminacy. The General Theory of Relativity is beginning of the changes. We have studied all aspects of this theory and established its wrong reasons in the works [16,17,26,38]. Unlike existing methods, our method does not use the distortion of mechanics. Besides for the solution of differential equations we have developed a new method of high ac- curacy. Therefore, our results are more accurate and reliable. But our work causes sharp objections of supporters of the existing methods. Below they are presented in the form of objections.

The success of scientific research often depends on the worldview of a scientist. He can have a misconception in the field, seemingly distant from the sphere of research. However, it is not to permit it to set scientific truth. The above applies to certain objections. We considered it necessary bring them and give them an answer, because this judgments interfere with the comprehension of scientific truth.

1. Objection. It is stated, paragraph 1. INTRODUCTION, that presently available methods for predicting the travel path of extraterrestrial objects lack sufficient accuracy..., but this pronouncement is not justified in any meaningful way. In fact, it is generally regarded that the limitation on prediction is set by observational uncertainties, not computational abilities. As is noted, the radiation pressure forces set a limit on prediction of Apophis and 1950 DA over very long periods of time, but again, the limitation is on our ability to measure or estimate these forces, not on computational limitations.

Answer. Apart from observational errors and the radiation-pressure force, there exist many other factors causing the difference between the calculated trajectory and the actual motion of an asteroid. In our paper, various approaches proposed by different authors are analyzed, and a method, free of many drawbacks, is used to solve the problem.

The referee expresses an opinion that in our paper we do not prove that methods capable of predicting the motion of asteroids with satisfactory accuracy are presently lacking. However, the absence of such methods immediately follows from the publications under consideration. It was not the point to prove that.

2. Objection. The suggestion to alter the orbits of these two objects to put them in orbit about the Earth seems absurd, and without justification. As noted, the change in velocity required to accomplish this is in the several $\mathrm{km} / \mathrm{sec}$ range. It is barely conceivable with present technology to make a change of a few $\mathrm{cm} / \mathrm{sec}$, five orders of magnitude less than would be required to place either object in Earth orbit. The authors make the cavalier statement that it might be possible to accomplish this, making reference to the advance from bare orbiting of instruments around the Earth to landing men on the moon in only a bit more than a decade. But they ignore the fact that the physics of how to do the latter was already known before the former was done, whereas in moving asteroids around by $\mathrm{km} / \mathrm{sec}$ increments of velocity is far beyond any currently understood technology. It's a bit like asking Christopher Columbus to plan a vessel to transport 400 people across the Atlantic in six 
hours - he wouldn't even know where to begin.

Answer. In our paper, we put forward an idea of capturing an asteroid in Earth orbit, analyze available possibilities in implementing this project, and calculate necessary parameter values.

We do not consider the engineering approaches that can be used in implementing the idea. That is a different field of knowledge, and this matter is to be analyzed in a separate publication.

As for the referee's remark on Christopher Columbus, the history saw how in 1485 the Columbus' proposal about an expedition to be send through the West Ocean to India was rejected by the Mathematician Council in Portugal. Later, in 1486, the project by Columbus was also rejected by the Academic Senate in the University of Salamanca, Spain, a famous university in the Middle Ages alongside with the Montpellier, Sorbonne and Oxford, because the project had incurred ridicule as resting on the "very doubtful" postulate of Earth's sphericity.

The objection expresses an opinion that the idea of man's exit into the outer space was implemented rather fast because the physics necessary for solving the problems behind that project was known, whereas the physics of how to implement our project presently remains an obscure matter.

The problem of man's exit into space was solved by engineers rather than scientists. When engineers had solved all problems, then established scientists had become able to catch the physical essence of the matter.

Presently, academic scientists are in captivity of relativistic fantasies about micro- and macro-world. As a result, they failed to properly understand the entire physical picture of our world, including the space travel physics. The best thing such physicists could do is not to interfere into the projects actually important for mankind like the project we discuss in our paper.

3. Objection. In considering the motion of the asteroids the paper describes only the asteroids are integrated, the other perturbations are derived from planetary ephemerides.

Answer. In our study, we integrated not only the motion of the asteroid; we also integrated the motion of other celestial bodies.

4. Objection. The integration method the authors present is not new (though the implementation in software may be). They present a simple, fixed-step Newtonian integrator that models only gravitational point masses. Far more sophisticated methods and physics have been published before precisely because the approach the authors go on to describe is inadequate.

Answer. Whether our integration method is new or not, the definition here is rather relative. Formula (2) in our paper gives a specialist the general idea behind our method and some details of its implementation is stated in the paper. Since none of the already existing methods was used in treating the problem we deal with in our study, we qualify our method as an original one. In our opinion, our method is akin to the method of TaylorSteffensen series rather than to the Newton method. In this approach the derivatives are determined by recurrence formulas. In our method (program Galactica) the derivatives are calculated under the exact analytical formulas, which we have deduced. This provides greater accuracy than other methods.

5. Objection. The presently available methods for predicting the travel path of extraterrestrial objects are fine. They are the same ones used to deliver spacecraft to planets and fit measurement data-arcs hundreds of years long.

Answer. One of the deficiencies of presently available methods for integrating the motion of celestial bodies is that those methods were constructed so that to provide a best fit to observational data. Within the period of available observations, those methods proved to yield rather good results. On the other hand, calculations of the motion of a previously unobserved object will obviously yield worse results. Also, calculations of the motion of a body observed during some observation period performed far outside this period will also yield less accurate results.

6. Objection. It is the limited knowledge of the physiccal properties of the objects that is the problem. Given measurements of those properties (spin, reflectivity, etc.), proper prediction is possible within computable error bounds.

Answer. The opinion that physical properties of an asteroid such as reflectivity or spin may notably affect the asteroid's motion is an erroneous opinion. This opinion is the consequence of deficiencies inherent to the methods mentioned in Answer 5. The actual motion of celestial bodies and spacecraft having been found different from their calculated motion, the people dealing with celestial mechanics undertook introducing additional fictitious forces into motion equations, such as the Yarkovsky force, whose magnitude was assumed to be dependent on the physical properties of a particular body under study.

7. Objection. It is stated (p. 1. INTRODUCTION), that “... the Apophis trajectory will for long remain... chaotic". No. Error growth is almost entirely in the alongtrack direction. It is not chaotic over relevant time-scales and measurements likely in 3 years will radically reduce those prediction uncertainties about $97 \%$. This is described in the papers the authors reference, so seems to be a misunderstanding.

Answer. It was the authors of cited publications rather than us that have qualified the motion of Apophis as a chaotic motion. 
8. Objection. The planetary ephemeris error it is far less than radiation related effects like solar pressure and Yarkovsky thermal re-radiation. The Giorgini et al. 2008 paper referenced shows what is required for better prediction is PHYSICAL KNOWLEDGE of the object (measurement), not METHOD.

Answer. Indeed, Giorgini et al. [4] have demonstrated that the solar pressure and Yarkovsky thermal re-radiation may have a considerable influence on the motion of celestial bodies. We, however, believe that the results by Giorgini et al. are erroneous. First, the forces of Giorgini et al. dealt with are fictitious nonexistent forces. Second, the interaction constants of those forces were artificially overestimated by Giorgini et al. [4].

We would like to deliver here some additional remarks concerning the fictitious nature of some forces. More than half a century ago it was shown by some physicists that no light pressure is observed in nature. Unfortunately, those results have been forgotten by many physicists.

The Yarkovsky force was introduced so that to compensate for the difference between the observed motion of celestial bodies, spacecrafts and their motion as predicted by contemporary theories. As it was already mentioned, the presently available methods for predicting the motion of celestial bodies suffer from serious deficiencies. Those deficiencies need to be overcome, and we believe that, following this, the difference between the actual motion of bodies and their motion as predicted assuming only the Newtonian gravity force to be operative will be made negligible and even exiled from final results. Then, additional fictitious forces will no longer be needed.

Let us give here some direct arguments proving that the forces under discussion are in fact fictitious forces. When in mechanics someone says that a force acts on a body, this does not mean that the force presents a material object. The sentence "a force acts on a body" is just slang. In mechanics, we imply that some body acts on another body. The influence is manifested in the changed motion of the second body. A change in motion is defined by body's acceleration. Hence, the action exerted by the first body consists in an acceleration experienced by the second body.

Man has invented mechanics in which actions are defined by an auxiliary quantity called the force. The force was defined as a quantity proportional to acceleration accurate to a factor (for details, see our books $[16,17]$ ).

So, the term "force" is not a name for an object in our world. When somebody says that a body on an inclined board experiences the actions due to the friction force and due to the gravity force, we imply that the body is acted upon by the board and, through the gravity interaction, by the Earth. When somebody says that the Moon is acted upon by the gravity force due to Earth, this means that it is the Earth that acts on the Moon.

On the other hand, in the case of light-pressure and Yarkovsky forces the acting bodies are missing. If one thinks of light considering it as a photon flux, he has to remember that photons have no mass, and they are therefore no physical bodies. Yarkovsky had invented his force as a force due to either particles, which are also nonexistent objects. Thus, both the light pressure and Yarkovsky thermal re-radiation are not actions due to bodies; such forces therefore bear no relation to mechanics. The only application fields of such forces are extra-sensory perception and Hollywood movies. Those forces "can be used" in ephemerid approximation models, such as SDM, because they all the same need to be fitted to many hundred thousand observations.

9. Objection. In p. 2. PROBLEM STATEMENT the insufficient information was provided to determine what integration algorithm was used by the authors. This is unacceptable given the rest of the paper.

The previously published literature on this subject is vast and highly developed and should be drawn upon and referenced.

Answer. Formulas (1) and (2) in our paper give the general idea behind our method, and they also define the form of master equations used in it. Details of the algorithm, and those of the method and equations, are too numerous to be outlined in the paper. We exploited our method over a period of more than ten years, and during that period, using the method, we have solved many problems. Some of our results were reported in publications [18-21,26,38]. In those publications, some details of the algorithm were described, and ample data on the adequacy of our method and credibility of solutions obtained, given. Below we list some of the problems that were tackled with the help of the Galactica software.

9.1. Evolution of planetary orbits and the orbit of Moon over the period of one hundred million years [18, 19]. It was for the first time that non-simplified differential equations of motion were integrated. The periods and amplitudes of planetary-orbit oscillations were evaluated, and stability of the Solar System was demonstrated (see Figures 3 and 4).

9.2. Optimal flight of a spacecraft to the Sun [21]. The spacecraft was proposed to use the gravitational maneuver near to Venus. The launch regime of the spacecraft allowing minimization of its starting velocity was identified.

9.3. Compound model of Earth rotation and the evolution of Earth rotation axis [20]. The Earth is considered as a system of several bodies located in the equatorial plane of a central body. The motion of one of the peripheral bodies models the motion of Earth rotation axis. The evolution of Earth rotation axis was calculated over a period of 110 thousand years. It was found that the Earth 
rotation axis precesses relative to the non-stationary axis of Earth orbital motion.

9.4. Compound model of Sun rotation and its outcomes for the planets [26] and [38]. The Sun rotation period is 25.38 days. The Galactica software was used to predict the outcomes of the compound model of Sun rotation on nearest planets. As a result of the calculations, an excessive revolution of Mercury perihelion was identified, which was previously explained assuming other mechanisms to be operative.

9.5. Multilayer ring structures [39]. The structure of interest comprises several rings, each of the rings involving several bodies. Evolution of several such ring structures was calculated, and stable and unstable configuretions were identified.

10. Objection. In p. 4. PREPARATION OF INITIAL DATA OF ASTEROIDS three pages of discussion and Equations (5)-(14) on the transformation of orbital elements to Cartesian coordinates could be deleted. This material is found in every introductory celestial mechanics course and need not be belabored.

Answer. Orbital elements can be transformed into Cartesian coordinates in different ways that yield different results. We have chosen the best transformation; we have deduced them and therefore give it in our paper. In addition, our consideration involves some formulas not be found in standard courses on celestial mechanics.

11. Objection. Further, the authors state their goal is to compute barycentric Cartesian coordinates, but then describe only heliocentric transformations. No information on if or how transformation from heliocentric to the barycentric needed by their code is given leads the reader to wonder if heliocentric coordinates were improperly used in the barycentric code.

Answer. The transformation of heliocentric coordinates to barycentric ones are firstly omitted from our paper as presenting a matter of common knowledge. Guided by the referee's remark, now we discuss it in our paper after Equation (14).

12. Objection. In end of p. 4. PREPARATION OF INITIAL DATA OF ASTREOIDS the authors have written: "... the masses of those bodies were modified by us..." This would introduce a dynamical inconsistency within the planetary ephemeris used to compute perturbations in the integration. Was the magnitude of this inconsistency computed?

The coordinates from DE405/406 said to be used are derived from the original planetary masses. Change those masses and the positions will change, hence perturbations on the object being integrated, hence the result of the integration.

Answer. We integrate Equations (1) for a total of twelve bodies, including the planets, Moon, Earth, and Apophis. We did not use planet and Moon coordinates taken from ephemerides; hence, any mass values can be adopted. The closer are the mass values to real masses, the better is the consistency between the calculated and observational data. We have checked this fact. In Galactica, the mass values and the initial data are specified in a separate file, which can easily be replaced with another file. Now, the relative mass values are taken from the DE405 system, whereas the absolute values have been recalculated as $G \cdot M_{\text {Earth, }}$, where $M_{\text {Earth }}$ is the Earth mass from the IERS system. The mass values adopted in our calculations are indicated in Table 2.

13. Objection. Studying Figure 5 at length, it is unable to interpret it. It seems to show two dots for Earth at point $A$; the text says there is only one.

Time scale would be better in calendar years instead of fractional centuries.

A figure is used if it shows relationships or trends clearly. This figure does not. Why not a useful table of numerical values?

Answer. The first dot in the horizontal line Ea refers to time $\mathrm{A}$. The second dot after interval $\Delta T=1$ year refers to the Earth, too. As it is seen from the graph, here the distance to which the asteroid closes the Earth is greater than $4.25 \times 10^{7} \mathrm{~km}$.

Figure 5 is indeed an uncommon representation. However, in case this uncommonness is overcome, Figure 5 gives a clear picture of the asteroid's approach to all the bodies over the whole considered time interval. No such picture can be grasped from a table.

14. Objection. It is stated in end of p. 5 APOPHIS ENCOUNTER WITH THE PLANETS AND THE MOON that "As for the possible approach of Apophis to the Earth in 2036, there will be no such approach..."

This is another fundamental misunderstanding of the paper resulting from an incorrect analysis.

The authors integrate a nominal orbit solution only and find it does not closely approach the Earth in 2036. However, it is necessary to examine not just the single nominal orbit, but the set of statistically possible orbit variations, defined by the orbit solution covariance matrix, as well as physical uncertainties (uncertainites).

The papers the authors cite go into such statistical approaches extensively.

Why does this fundamental issue of modern orbit determination not exist in this paper?

The analysis the authors provide does not recognize the statistical nature of the problem. The authors approach is not acceptable for analyzing such problems because it ignores the statistical distribution of orbit variations defined by the measurement dataset.

This alone renders the paper and its conclusions irrelevant to readers.

Answer. We regard such a statistical study a vain undertaking. 
If many measurement data for a parameter are available, then the nominal value of the parameter, say, eccentricity $e_{n}$, presents a most reliable value for it. That is why a trajectory calculated from nominal initial conditions can be regarded as a most reliable trajectory. A trajectory calculated with a small deviation from the nominal initial conditions is a less probable trajectory, whereas the probability of a trajectory calculated from the parameters taken at the boundary of the probability region (i.e. from $e=e_{n} \pm \sigma_{e}$ ) tends to zero. Next, a trajectory with initial conditions determined using parameter values trice greater than the probable deviations (i.e. $e=e_{n} \pm$ $3 \sigma_{e}$ ) has an even lower, negative, probability. Since initial conditions are defined by six orbital elements, then simultaneous realization of extreme (boundary) values ( \pm $3 \sigma$ ) for all elements is even a less probable event, i.e. the probability becomes of smaller zero.

That is why it seems that a reasonable strategy could consist in examining the effect due to initial conditions using such datasets that were obtained as a result of successive accumulation of observation data. Provided that the difference between the asteroid motions in the last two datasets is insignificant over some interval before some date, it can be concluded that until this date the asteroid motion with the initial conditions was determined quite reliably.

Such computations were carried out and described in the additional Section 6. Influence of initial conditions.

15. Objection. In p. 6. APOPHIS ORBIT EVOLUTION the authors describe integrating the orbit of Apophis over 200 years, writing out a file of coordinates each year. They then go back and, starting from each file, integrate one Apophis orbit period and save that to a file.

Why? 201 integrations are being done when one would suffice. Is not going back and integrating from the starting point of each yearly file the same as integrating continuously over the span?

Answer. On integration of Equation (1), we obtain coordinates of each body in the barycentric system. For determining a body's orbital elements, it is required to consider the coordinates of the body with respect to a parent body (for an asteroid, with respect to the Sun) during one orbital period. To avoid a complex logic in choosing coordinate values, in integration over the whole time interval of interest, we chose to adhere to the strategy described in the paper.

16. Objection. In p. 9. POSSIBLE USE OF ASTEROID APOPHIS the argument made for capturing Apophis into Earth orbit is at a level suitable for sketching on a napkin. No discussion of material properties, or mechanics. The composition of Apophis is unknown and the discussion amounts to speculation for personal entertainment.

Answer. In the paper, we describe available strategies for making the asteroid an Earth-bound satellite and cal- culate parameter values necessary for realization of such a project. An analytical background behind those strategies is developed. The motion of the asteroid after trajectory correction and the motion of formed satellites were determined by integrating Equations (1). We do not describe all the obtained results in our paper; however, those results were used to substantiate the proposed strategies in capturing the asteroid in Earth orbit. Those strategies are unobvious, and it should be remembered that one can propose strategies that never can be implemented. We propose realizable strategies. We have calculated the orbit evolution of the satellites and proved that those orbits can be made stationary for a long time. The computations for satellites were made taking into account the action exerted on them by all bodies. We believe our calculations to be original. Following our publication, other workers will move farther in this direction.

How can those strategies be implemented? This matter will be discussed after the present results are reported in the literature. For the time being, we raise the issue of making an asteroid an Earth-bound satellite. This issue is given rather a deep analysis. All computations are performed at a good scientific level. That is why our results are not to be ignored, and the work, regarded as a sketch on napkin, to be one day thrown away. It is more probable that it is the statistical data on the asteroid's encounter with the Earth rather than our paper that will be one good day thrown away.

17. Objection. Ii is stated in the beginning p. 9 that "Over subsequent 1000 years, Apophis will never approach our planet closer". The analyses given cannot support the statement. All uncertainties physical and measurement are ignored by the authors. Only the single nominal orbit is considered. This is unacceptable and the results of no interest to readers.

Answer. Indeed, our calculations show that the asteroids will not hit the Earth. On conscientious analysis, statistical data on such collisions in the cited publications are also indicative of this fact. Only undisguised tricksters, reasoning from such statistics, can frighten the society with the threat of Apophis danger. With passage of time, people usually become aware of scientific trickery, and this deteriorates their trustfulness to science. The way we propose in our paper will allow mankind to develop in the future a good method for preventing the potential threat of asteroid's collisions with Earth. Note that such method can only be implemented if we find a way for making asteroids Earth-bound satellites.

The dynamics of Solar system is not linear. Thus, there can be orbits with initial conditions intermediate to those that authors used, which can lead to closer approaches. Actually, the theory predicts that there are KEYHOLES, associated to RESONANT RETURNS which can lead to 
collisions. This aspect is missing in this work.

In this paper no new results. This looks more like the report of a beginner entering in this field for the first time, and just setting up the software tools and the conceptual know-how to be able, in the future, to perform research in this field. In particular some conceptual building blocks are still missing, such as the notion of chaos (mentioned just once as dreaded possibility, while it is a well established fact that all the asteroids which can impact the Earth are on chaotic orbits), and the effect of nonlinearity in the orbit determination and in the propagation of the uncertainty to a future time.

Answer. There are three aspects in this objection:

1) Within the uncertainty ellipsoid of the orbital elements do not found a collision with Earth;

2) Do not shown the KEYHOLES of resonant and chaotic trajectories;

3) There are no new results.

Our paper shows that the search for collisions within the uncertainty ellipsoid of the orbital elements is meaningless work. We have also shown that the conclusion about chaotic motion is caused by imperfection of methods of integrating the equations.

By another method and in another way we are solving this problem. We have got new results: Asteroids Apophis and 1950 DA do not impact the Earth. In addition we are putting forward and are based the new idea: The transformation of the asteroids in the satellites.

So, in our paper the new methods are used, the new results are received and the new ideas are putted forward.

In contrast to our paper the published papers, which we cited above, prove the false idea about collisions in 2036 and in 2880 . These papers are misleading readers. When the scientists' errors become clear, the society has intensified distrust of science.

In published papers the imaginary constructs are investigated: Chaos, resonances, keyholes, etc. Their authors use methods with the imaginary precision by which supposedly can determine the motion of the planets up to $\mathrm{mm}$ and up to marcsec. We emphasize the imaginary precision that arises when comparing the methods on those observations, to which they are fitted. If someone is using them to calculate the outside of this area, the motions of bodies differ significantly from the calculated movements. The authors of published papers believe that there are fictitious forces (Yarkovsky force, etc.), resonances and keyholes, which make the body motion chaotic. That is, rather than to doubt the accuracy of the methods they put forward the reasons for their justification.

The same methods found that the Solar system after 20 million years ago is starting to change, and in the future because of the chaos it begins to collapse. The reason for these phenomena lies in the imperfection of methods for calculating the motions. In contrast, our method allowed us to integrate the equations of the Solar system motion for 100 million years: The Solar system is stable and no signs of change. So the keyholes, resonances, chaos and the fictitious forces appear due to imperfect methods of calculating the motions.

Our paper cannot be viewed superficially, it must be deeply studied. It gives a lot of new knowledge about the evolution of the asteroids motion, the accuracy of integration methods and on the ways in which to develop these methods.

The modern celestial mechanics dominates by ideas of indeterminacy, of unpredictable resonances and of chaotic motions. Our paper provides the mathematical tools and techniques that allow us to calculate the movement with known accuracies, and then to implement them. The paper presents a path that each can go through and check out our results. This is the science.

But the chaos, the resonances, the keyholes are not the science, those are Extrasensory.

It is need return to the classical celestial mechanics, the creators of which are not doubted the determinacy of movements.

\section{Conclusions}

1) The instability and randomness in dynamics of planets and asteroids is caused by imperfection of methods of account of movements;

2) The parameters of planets orbits are steady changing with the certain periods and amplitudes;

3) On 21 hour 45' GMT, April 13, 2029 Apophis will pass close to the Earth, at a minimum distance of 6 Earth radii from Earth's center. This will be the closest pass of Apophis near the Earth in the forthcoming one thousand years;

4) Calculations on making Apophis an Earth bound satellite appropriate for solving various space exploration tasks were performed;

5) The asteroid 1950 DA will twice approach the Earth to a minimal distance of 2.25 million $\mathrm{km}$, in 2641 and in 2962;

6) At any epoch, the asteroid 1950 DA can be made an Earth-bound satellite by increasing its aphelion velocity by $\sim 1 \mathrm{~km} \cdot \mathrm{s}^{-1}$ and by decreasing its perihelion velocity by $\sim 2.5 \mathrm{~km} \cdot \mathrm{s}^{-1}$.

\section{Acknowledgements}

The authors express their gratitude to T. Yu. Galushina and V. G. Pol, who provided them with necessary data on asteroid Apophis. They are also grateful to the staff of the Jet Propulsion Laboratory, USA, whose sites were used as a data source from which initial data for integration of motion equations were borrowed. The site by 
Edward Bowell (ftp://ftp.lowell.edu/pub/elgb/) was helpful in grasping the specific features of asteroid data representation and in avoiding possible errors in their use. Krotov O. I. took part in calculations of the Apophis motion on the system Horizons. The calculations were carried out on the supercomputer of the Siberian Supercomputer Centre of Siberian Branch RAS.

The study was carried out as part of Integration Programs 13 (2008-2011) of the Presidium of the Russian Academy of Sciences.

\section{REFERENCES}

[1] J. Laskar, "Large-Scale Chaos in the Solar System," Astronomy \& Astrophysics, Vol. 287, No. 1, 1994, pp. L9L12.

[2] J. Laskar, P. Robutel, F. Joutel, M. Gastineau, A. C. M. Correia and B. Levrard, "A Long-Term Numeriucal Solution for the Insolation Quantities of the Earth," Astronomy \& Astrophysics, Vol. 428, No. 1, 2004, pp. 261-285. doi:10.1051/0004-6361:20041335.

[3] J. Laskar, A. C. M. Correia, M. Gastineau, F. Joutel, B. Levrard and P. Robutel, "Long Term Evolution and Chaotic Diffusion of the Insolation Quantities of Mars," Astronomy \& Astrophysics, Vol. 428, No. 1, 2004, pp. 261285. doi:10.1051/0004-6361:20041335.

[4] J. D. Giorgini, L. A. M. Benner, S. I. Ostro, H. C. Nolan and M. W. Busch, "Predicting the Earth Encounters of (99942) Apophis," Icarus, Vol. 193, No. 1, 2008, pp. 119. doi:10.1016/j.icarus.2007.09.012.

[5] R. Tucker, D. Tholen and F. Bernardi, MPS 109613, Minor Planet Center, Cambridge, 2004. http://www.minorplanetcenter.net/db_search

[6] G. J. Garradd, MPE Circ., Minor Planet Center, Cambridge, 2004, Y25.

http://www.minorplanetcenter.net/iau/mpec/K04/K04Y25. html

[7] L. V. Rykhlova, B. M. Shustov, V. G. Pol and K. G. Sukhanov, "Urgent Problems in Protecting the Earth against Asteroids," Proceedings of International Conference NearEarth Astronomy, Nalchik, 3-7 September 2007, pp. 2533.

[8] V. A. Emel'yanov, Y. K. Merkushev and S. I. Barabanov, "Cyclicity of Apophis Observation Sessions with Space and Ground-Based Telescopes," Proceedings of International Conference Near-Earth Astronomy, Nalchik, 3-7 September 2007, pp. 38-43.

[9] V. A. Emel'yanov, V. I. Luk'yashchenko, Y. K. Merkushev and G. R. Uspenskii, "Determination Accuracy of Asteroid Apophis' Orbit Parameters Ensured by Space Telescopes," Proceedings of International Conference NearEarth Astronomy, Nalchik, 3-7 September 2007, pp. 5964.

[10] L. L. Sokolov, A. A. Bashakov and N. P. Pit'ev, "On Possible Encounters of Asteroid 99942 Apophis with the Earth," Proceedings of International Conference NearEarth Astronomy, Nalchik, 3-7 September 2007, pp. 3338.
[11] E. Everhart, "Implicit Single-Sequence Methods for Integrating Orbits," Celestial Mechanics and Dynamical Astronomy, Vol. 10, No. 1, 1974, pp. 35-55.

[12] L. E. Bykova and T. Y. Galushina, "Evolution of the Probable Travel Path of Asteroid 99942 Apophis," Proceedings of International Conference Near-Earth Astronomy, Nalchik, 3-7 September 2007, pp. 48-54.

[13] L. E. Bykova and T. Yu. Galushina, "Fundamental and Applied Problems in Modern Mechanics," Proceedings of the 6th All-Russia Scientific Conference, Tomsk, 30 September-2 October 2008, pp. 419-420.

[14] E. A. Smirnov, "Advanced Numerical Methods for Integrating Motion Equations of Asteroids Approaching the Earth," Proceedings of International Conference NearEarth Astronomy, Nalchik, 3-7 September 2007, pp. 5459.

[15] V. V. Ivashkin and K. A. Stikhno, "An Analysis of the Problem on Correcting Asteroid Apophis' Orbit," Proceedings of International Conference Near-Earth Astronomy, Nalchik, 3-7 September 2007, pp. 44-48.

[16] J. J. Smulsky, “The Theory of Interaction," Publishing House of Novosibirsk University, Novosibirsk, 1999.

[17] J. J. Smulsky, "The Theory of Interaction," Publishing House Cultural Information Bank, Ekaterinburg, 2004.

[18] E. A. Grebenikov and J. J. Smulsky, "Numerical Investigation of the Mars Orbit Evolution in the Time Interval of Hundred Million," A. A. Dorodnitsyn Computing Center of RAS, Moscow, 2007.

[19] V. P. Melnikov and J. J. Smulsky, “Astronomical Theory of Ice Ages: New Approximations. Solutions and Challenges," Academic Publishing House, Novosibirsk, 2009.

[20] V. P. Mel'nikov, I. I. Smul'skii and Y. I. Smul'skii, “Compound Modeling of Earth Rotation and Possible Implications for Interaction of Continents," Russian Geology and Geophysics, Vol. 49, No. 11, 2008, pp. 851-858. doi:10.1016/j.rgg.2008.04.003.

[21] J. J. Smulsky, "Optimization of Passive Orbit with the Use of Gravity Maneuver," Cosmic Research, Vol. 46, No. 55, 2008, pp. 456-464. doi:10.1134/S0010952508050122.

[22] K. F. Steffensen, "On the Problem of Three Bodies in the Plane," Kongelige Danske Videnskabernes Selskab, Matematisk-Fysiske Meddelelser, Vol. 13, No. 3, 1957.

[23] M. S. Yarov-Yarovoi, "The Use of Refined Methods of Numerical Integration in Celestial Mechanics," GAISh Transactions, Vol. 45, 1974, pp. 179-200.

[24] J. J. Smulsky, "The Axisymmetrical Problem of Gravitational Interaction of N Bodies," Matematicheskoe Modelirovanie, Vol. 15, No. 5, 2003, pp. 27-36.

[25] T. R. Quinn, S. Tremaine and M. Duncan, "A Three Million Year Integration of the Earth's Orbit," Astronomical Journal, Vol. 101, 1991, pp. 2287-2305.

[26] J. J. Smulsky, "New Components of the Mercury's Perihelion Precession," Natural Science, Vol. 3, No. 4, 2011, pp. 268-274. doi:10.4236/ns.2011.34034.

[27] S. Newcomb, "The Elements of the Four Inner Planets and the Fundamental Constants of Astronomy," Govern- 
ment Printing Office, Washington DC, 1895.

[28] J. L. Simon, P. Bretagnon, J. Chapront, G. Francou and J. Laskar, "Numerical Expression for Precession Formulae and Mean Elements for the Moon and the Planets," Astronomy \& Astrophysics, Vol. 282, 1994, pp. 663-683.

[29] E. Bowell, "The Asteroid Orbital Elements Database," 2008. ftp://ftp.lowell.edu/pub/elgb

[30] California Institute of Technology, "JPL Small-Body Database 2008. Jet Propulsion Laboratory. 99942 Apophis (2004 MN4)," 2008. http://ssd.jpl.nasa.gov/sbdb.cgi?sstr=Apophis;orb=1

[31] G. N. Duboshin, "Celestial Mechanics and Astrodynamics," Nauka, Moskva, 1976.

[32] J. J. Smulsky, "A Mathematical Model of the Solar System," In: V. A. Bereznev, Ed., The Theoretical and Applied Tasks of the Nonlinear Analysis, A. A. Dorodnicyn Computing Center, Moscow, 2007, pp. 119-139.

[33] "Ephemerides of the Jet Propulsion Laboratory," 2008. http://ssd.jpl.nasa.gov/?ephemerides

[34] E. M. Standish, "JPL Planetary and Lunar Ephemerides," Interoffice Memorandum, 1998.

[35] J. D. Giorgini, et al., "Asteroid 1950 DA Encounter with Earth in 2880: Physical Limits of Collision Probability Prediction," Science, Vol. 296, No. 5565, 2002, pp. 132136.

[36] U. Corliss, "Mystery of the Universe," Mir, Moscow, 1970.

[37] B. D. V. Cole and D. W. Cox, "Islands in Space," Chilton Books, Philadelphia, 1964.

[38] J. J. Smulsky, "Gravitation, Field and Rotation of Mercury Perihelion," Proceedings of 15th Annual Conference of the Natural Philosophy Alliance, Albuquuerque, 7-11 April 2008, pp. 254-260.

[39] I. I. Smul'skii, "Multilayer Ring Structures," Physics of Particles and Nuclei Letters, Vol. 8, No. 5, 2011, pp. 436440. doi:10.1134/S1547477111050189. 